\title{
Holographic in situ stress measurements
}

\author{
Jay D. Bass ${ }^{\star}$, Douglas Schmitt and Thomas J. Ahrens
}

Seismological Laboratory 252-21, California Institute of Technology, Pasadena, CA 91125, USA

Accepted 1985 August 14. Received 1985 August 14; in original form 1984 August 26

Summary. A new instrument for measuring the in situ level of stress in boreholes has been developed. The instrument operates on the principle of locally relieving the stresses acting on a rock mass by drilling a small hole into the borehole surface and recording the resultant displacement field by holographic interferometry. Because the recording technique is optical, the entire displacement field due to stress relief is obtained. A description of the stressmeter, theory of the interferometric technique, data reduction methodology, and results of laboratory stress relief calibration tests are presented. In addition, we present results from a field deployment of the instrument in an underground shale mine in Garfield County, Colorado using a test borehole within a support pillar. Sufficient data were obtained to constrain five of six stress components at a shallow level of the test borehole, thereby demonstrating the viability of the holographic technique. The holographic stressrelief data yield an approximate EW maximum horizontal stress direction. By comparison with previous hydrofracture measurements of Bredehoeft et al., our results indicate substantial stress-relief near the pillar face, thus masking any relicts of the far-field tectonic stress.

Key words: holography, stress

\section{Introduction}

Knowledge of the in situ stress field in the Earth's crust is important to our understanding of numerous geophysical phenomena, such as the driving mechanisms and regimes of plate tectonic processes. Of specific interest are the stresses acting on the lower boundary of the lithosphere and on the lateral boundary of plates (Richardson, Solomon \& Sleep 1979; Solomon, Richardson \& Bergman 1980; Hager \& O'Connell 1981; McGarr, Zoback \& Hanks 1982 ), and the stresses associated with various stages of mid-ocean ridge volcanism, volcanism associated with subduction, and intraplate volcanism (Smith \& Bailey 1968;

${ }^{\star}$ Present address: Department of Geology, University of Illinois, Urbana, IL 61801, USA. 
Nakamura \& Uyeda 1980). The geotechnical applications of in situ stress data range from design of underground openings (Jaeger \& Cook 1979; Haimson 1975), to the exploitation, via controlled hydrofracture, of petroleum resources (Simonsen, Abou-Sayed \& Clifton 1978), and the storage of waste in the Earth (Doe et al. 1983).

A new apparatus for measuring in situ stress in boreholes is described in the present paper. Our motivation for developing the apparatus stems from:

(1) A need to create an instrument which yields essentially the same information as hydrofracture (Zoback \& Haimson 1983) or the triaxial strain cell (Leeman \& Hayes 1966), but which is not restricted to operate near the surface within the range of overcoring, and can measure both the vertical and effective horizontal stresses surrounding a single borehole.

(2) To develop an instrument which can be used in existing drillholes and does not require a drilling rig to operate, but rather can be deployed from a portable wireline hoist. Moreover, it is highly desirable that the stress-measuring apparatus be able to make a measurement in a short period of time so that a large amount of data may be obtained in a cost-effective way.

(3) To develop a technique that can operate in boreholes but need not require the conditions of sufficiently high downhole pore pressure and sufficiently low permeability to allow hydrofracture.

This paper describes the final design of a $29 \mathrm{~cm}$ diameter in situ stressmeter which utilizes the technique of holographic interferometry for recording data, and satisfies most of the criteria listed above. The current version of the instrument has evolved from earlier designs reported by Schmidt et al. (1974), Ahrens, Jacoby \& Bhuta (1975), Cohn \& Ahrens (1982), and Cohn (1983). In addition, we outline below the theoretical basis for the measurement technique, details of several laboratory calibration tests, the methodology employed in reducing and interpreting holographic data, and the results of a field deployment of the instrument in a horizontal borehole.

\section{Description of the instrument and principle of operation}

The instrument we have developed utilizes a stress relief method for deducing the state of stress in a borehole, and is conceptually similar to conventional overcoring methods (e.g.
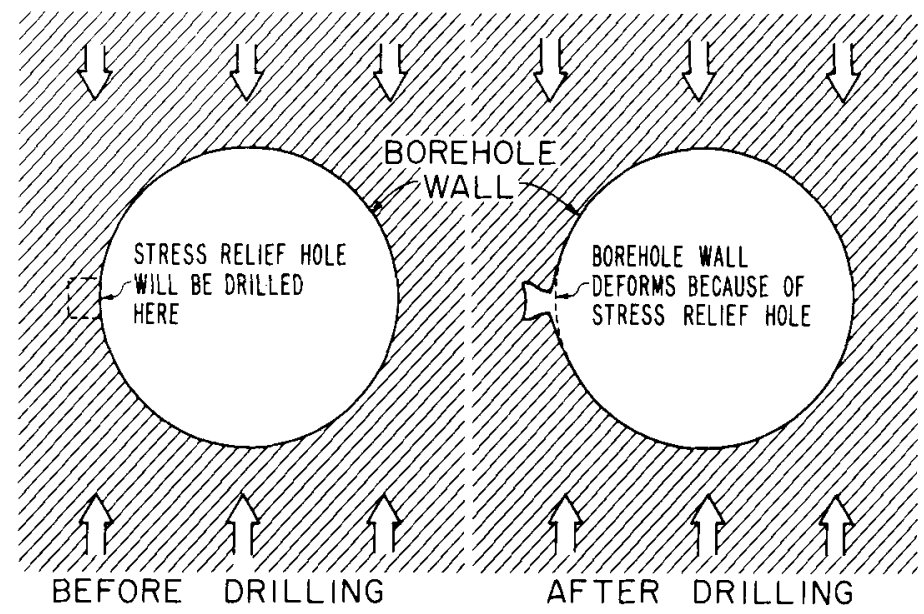

Figure 1. Cross-section of a borehole under a far-field uniaxial stress, illustrating the stress-relief method employed to measure stress. On the left the borehole surface is undisturbed. A cylindrical stress-relief hole is drilled into the borehole (right), and the adjacent borehole surface deforms to a degree that depends upon the stress level. The stressmeter records the displacement field holographically. 
Leeman \& Hayes 1966). Consider a borehole within a stressed rock mass. If a small cylindrical 'side-core' hole is drilled normal to the wall of the borehole, the stresses acting on the rock will be locally relieved (Fig. 1). As a result there is an elastic deformation, or relaxation of material in the vicinity of the stress-relieving hole. The magnitude of the displacement will depend, among other things, upon the size of the stress-relieving role, the state of stress on the rock, and both the elastic and anelastic properties of the rock. By measuring the deformation about the stress-relief hole the components of stress acting on the rock may be deduced, assuming that the elastic moduli of the rock are known and that anelastic deformation is negligible. In fact, three sets of strain measurements, at properly chosen azimuths, are sufficient to obtain the complete far-field state of stress at a given depth. We will elaborate on this point in a later section.

One of the unique features of our instrument is that displacements on the borehole wall are recorded optically as an interference hologram on a piece of photographic film. For the moment, let it suffice that a hologram is a 'three-dimensional-picture' of an object; a more complete description of the holographic process is described below. Holographic interferometry is an extremely sensitive method of recording small displacements and, in the field of rock mechanics, has previously been used for monitoring the deformation of rock samples in laboratory experiments (Granryd, Getting \& Spetzler 1983; Kurita et al. 1983; Spetzler, Getting \& Martin 1979; Spetzler \& Martin 1974). In the present application, an interference hologram is produced by recording holograms of the borehole surface in both the undisturbed and deformed states on the same piece of film. Any deformation occurring between exposures of the two holograms gives rise to a series of dark lines, or fringes, on the holographic image of the object, and each fringe may be related to a magnitude of displacement. Each interferogram gives a complete description of the displacement field of the borehole surface about the stress-relieving hole, and this is a distinct advantage over electromechanical devices, such as strain gauges, which yield only measurements of local displacement over a small area.

From the above brief overview of our technique, it is clear that the two main operations

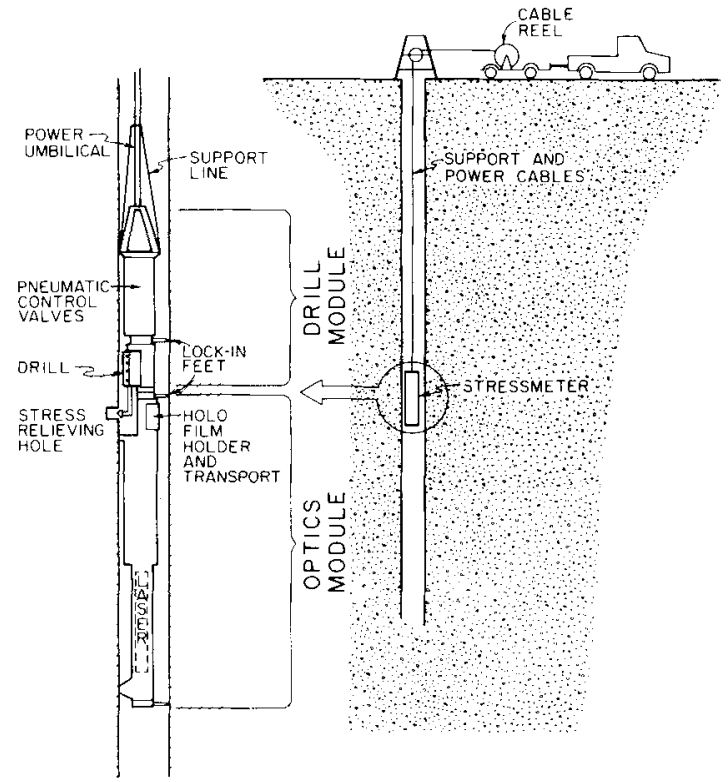

Figure 2. Diagram of the entire stressmeter locked into a borehole. 
associated with each measurement are the drilling of a stress-relief hole and the recording of a pair of holographic images. The instrument is accordingly divided into two sections, as shown in Fig. 2, each concerned with performing one of the two main functions, and which are referred to as the drill and optics modules. The use of holographic interferometry as a means of recording displacements imposes rather severe requirements on the design of the instrument, most having to do with physical and mechanical stability during a measurement. Therefore, in order to understand the various components and construction of the instrument, it is useful to outline briefly the basic theoretical concepts of holography. More complete discussions of the theory and applications of holography may be found in numerous books and review articles (e.g. Waters 1974; Vest 1979).

\subsection{HOLOGRAPHIC THEORY}

Holography is a method for recording both the phase and amplitude information in an optical wavefront on a photographic medium. It was first invented by Gabor $(1948,1949$, 1951) but did not become a practical technique until invention of the laser provided a suitably coherent light source. When light reflected from an object is holographically recorded, the optical wavefront can later be reconstructed by proper illumination, producing an identical image which has a three-dimensional quality to it. The 3-D quality of holograms is possible because the phase of the original wavefront is preserved, and it is this characteristic which distinguishes holography from conventional photography, where only the intensity of light is recorded.

The principles of holography may be illustrated by the experimental apparatus shown in Fig. 3(a). This figure illustrates all of the basic elements needed to produce a two-beam, or off-axis hologram of the type devised by Leith \& Upatnieks $(1962,1964)$. This is a widely used type of holographic method and is incorporated into our stress-measuring apparatus. Highly coherent, monochromatic light from a laser is divided into two beams by a beam-

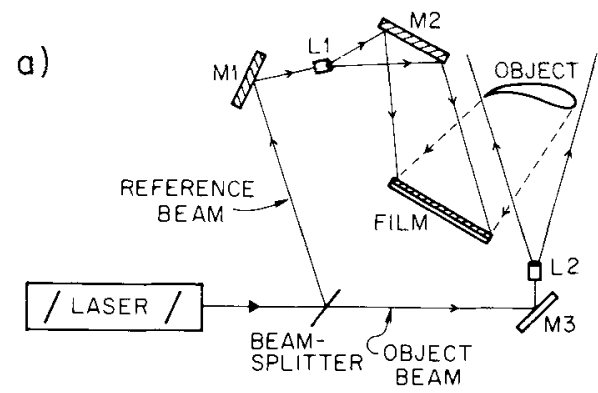

b)

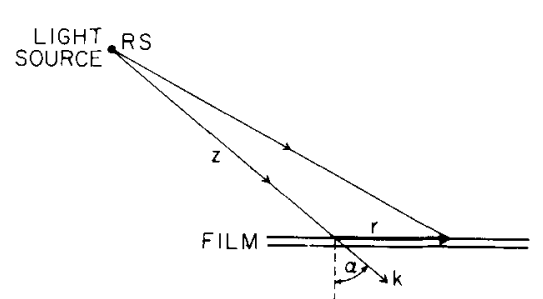

Figure 3. (a) A typical experimental apparatus for recording a Leith-Upatnieks, or two-beam, hologram. $M 1, M 2$ and $M 3$ are mirrors; $L 1$ and $L 2$ are expanding lenses. (b) Light from source RS with wavevector $k$ striking a film at angle $\alpha$. The light at position $\mathbf{r}$ will be phase shifted due to the angle $\alpha$ between $\mathbf{k}$ and the film plane, and the curvature of the wavefront (see equation 2). 
splitter. One of the beams is used to illuminate the object and is thus called the object beam, while the other, called the reference beam, is directed at the holographic film without hitting the object. The complex amplitude of these two monochromatic wavefronts of light may be represented in the following form:

$U_{\mathrm{o}}(\mathrm{r})=A_{\mathrm{o}}(\mathrm{r}) \exp i\left[\phi_{\mathrm{o}}(\mathbf{r})+\omega t\right]$

$U_{\mathrm{r}}(\mathbf{r})=A_{\mathrm{r}}(\mathbf{r}) \exp i\left[k\left(r \sin \alpha+r^{2} / 2 Z\right)+\omega t\right]$

where the subscripts o and r refer to the object and reference beams, respectively (Waters 1974). Each light wave is described by an amplitude $A$, phase $\phi$ and angular frequency $\omega$. The term $k\left(r \sin \alpha+r^{2} / 2 Z\right)$ in the expression for the reference wave accounts for changes in phase due to the angle $\alpha$ between $k$ (wave vector $=2 \pi /$ wavelength) and the film plane normal, and also for curvature of the wavefront (Fig. 3b). At the film surface the resultant wave amplitude $U$ is the sum $U_{\mathrm{o}}+U_{\mathrm{r}}$. The film will record the intensity of light $I$, which is given by:

$I=U U^{*}=A_{\mathrm{o}}^{2}+A_{\mathrm{r}}^{2}+2 A_{\mathrm{o}} A_{\mathrm{r}} \cos \left[\phi_{\mathrm{o}}(r)-k\left(r \sin \alpha+r^{2} / 2 Z\right)\right]$

where $U^{*}$ is the complex conjugate of $U$. Thus, interference of the reference and object waves at the film surface leads to a variation in the recorded light intensity as described by the cosine term in equation (3). Development of the photographic plate results in a collection of very finely spaced $\left(\sim 1200\right.$ lines $\left.\mathrm{mm}^{-1}\right)$ light and dark areas, or fringes, on the film. If the developed film is placed in its original position and illuminated only by the reference beam, the fringes on the film will act as a diffraction grating which serve to reconstruct the original object beam wavefront. The exposed and developed holographic film does not look anything like the recorded object, as in the case of a conventional photographic negative; rather it is the fringes, closely spaced and normally unobservable, which carry all of the information about the recorded object, and a reconstruction beam is needed to recover the original scene from the hologram.

Holographic images may actually be reconstructed in two ways, as shown in Fig. 4. A virtual image is produced by illuminating the hologram in a way that duplicates the geometry of the film and reference beam during exposure. Alternatively, a real image can be reconstructed by illuminating the hologram with the conjugate to the original reference beam. A conjugate beam is one that is opposite in direction of propagation and curvature to the original reference beam. The practical difference between the virtual (orthoscopic) and real (pseudo-scopic) images are that the virtual image is a true likeness of the object that may

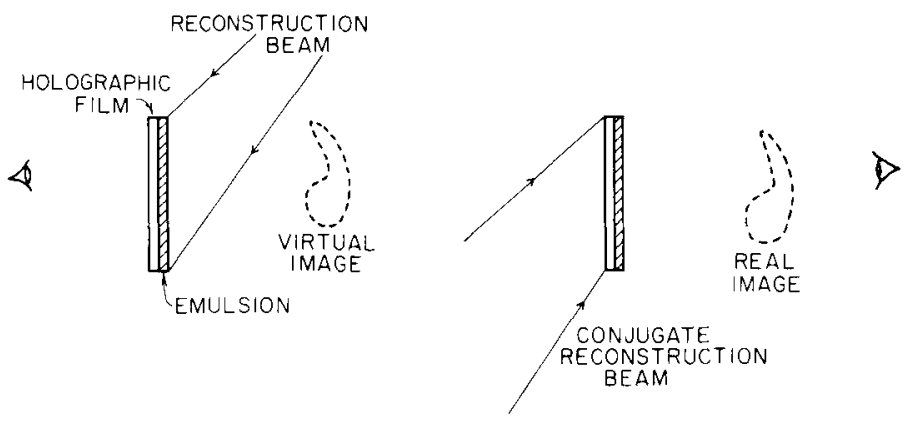

Figure 4. Reconstruction of a Leith-Upatnieks hologram. On the left, the reconstruction beam is identical to the original reference beam, whereas the reconstruction beam in the right part of the figure is conjugate to the original reference beam. 
be easily observed with the eye or photographed with a camera, whereas the real image is inverted and difficult to observe visually but may be conveniently projected on to a screen. All of the photographs of holograms shown in this paper (i.e. Plate 1) were produced using light directly from a laser as a conjugate beam. The reconstructed image was photographed by projecting it on to a sheet of film and recording it as a conventional photographic negative.

In order to record a two-beam hologram successfully several conditions must be satisfied. The light source must be highly coherent. Temporal coherence is related to the bandwidth of the source and may be measured in terms of a coherence length, or that length over which successive wavefronts have a consistent relationship. Lasers are a source of very nearly monochromatic light and therefore have large coherence lengths, on the order of several tens of centimetres, whereas white light is incoherent. If light from the reference and object beams are not coherent, then interference of the two beams will not produce interferences fringes of sufficiently high contrast to record a hologram. Therefore, the difference in the path length (from the beamsplitter in Fig. 3a to the holographic film) for the object and reference beams must be less than the coherence length of the source $(\sim 20-30 \mathrm{~cm})$. In addition, it is important that components of the holographic recording apparatus remain fixed relative to one another during an exposure. Motions of the order of a fraction of a wavelength of the light source will severely compromise the holographic fringe contrast and may make the recording of an intelligible hologram impossible.

Almost since its inception as a practical tool, holography has been used to detect small motions, deformations or vibrations by the technique of holographic interferometry (e.g. Horman 1965; Powell \& Stetson 1965; Haines \& Hildebrand 1966; Heflinger, Wuerker \& Brooks 1966). The particular technique used in our stressmeter is double-exposure holographic interferometry and may be understood as a direct extension of the holographic method so far discussed. Consider an object whose image has been recorded on a holographic plate. If the object is slightly deformed in some way without disturbing any other part of the recording apparatus, and another hologram is then made of the object on the same photographic plate, the reconstructed wavefronts of the two separate holographic exposures will themselves interfere. On the resulting hologram it will appear as if the object were recorded in both states at the same time. Consider further the path lengths of light reflected by an arbitrary point on the object to a position on the film plane, both before and after the deformation. If the difference in these path lengths is an even number of half wavelengths there will be no discernible effect of the deformation on the recorded or reconstructed light intensity (assuming, of course, small deformations). On the other hand, if the path length difference is an odd number of half wavelengths, light reflected from the point in the two states will be out of phase and cancel. Therefore, the reconstructed image from the double exposure hologram the object will have a series of bright and dark fringes superimposed upon it. These displacement interference fringes should not be confused with the fine diffraction-grating-type fringes which form the actual hologram. Each interference fringe is a contour of constant phase difference of the reflected light before and after deformation, and the fringe pattern resulting from a given deformation may be obtained in a straightforward manner.

An example of an interference hologram, or interferogram, is shown in Plate 1. The object is an aluminium plate with a throughgoing hole subjected to a horizontal uniaxial stress between holographic exposures. It is immediately clear from the fringe pattern that both the stress and strain are concentrated about the hydraulic ram producing the stress. In addition, this hologram shows evidence for a component of uniform horizontal translation parallel to the film plane. Thus, this deceptively simple fringe pattern proved to be difficult 
to analyse theoretically and led to our designing a better apparatus for uniaxially stressing samples. Nonetheless, Plate 1 demonstrates in a clear and dramatic way how the deformation of a sample is recorded by double exposure holographic interferometry.

\subsection{OPTICS MODULE}

All of the components necessary to record holograms of the borehole surface are contained within the optics module, a detailed cross-section of which is shown in Fig. 5. Light from a 5 mW He-Ne laser (Spectra Physics Model 159) is admitted to the camera section, containing the holographic recording apparatus, via an electronic shutter, $\mathrm{S}$. The laser compartment and camera section are isolated by a glass plate and aluminium bulkhead to prevent air convection from disturbing the optical components during an exposure. Within the camera section the optics are arranged, with minor modifications, as described by Schmidt et al. (1974). The laser light is divided into a reference beam $\mathrm{R}$ and object beam $\mathrm{O}$, by a beamsplitter consisting of a glass plate thick enough to allow isolation of the front surface reflection from any ghosts. The reference beam is expanded by a lens, $\mathrm{L}$, and directed on to
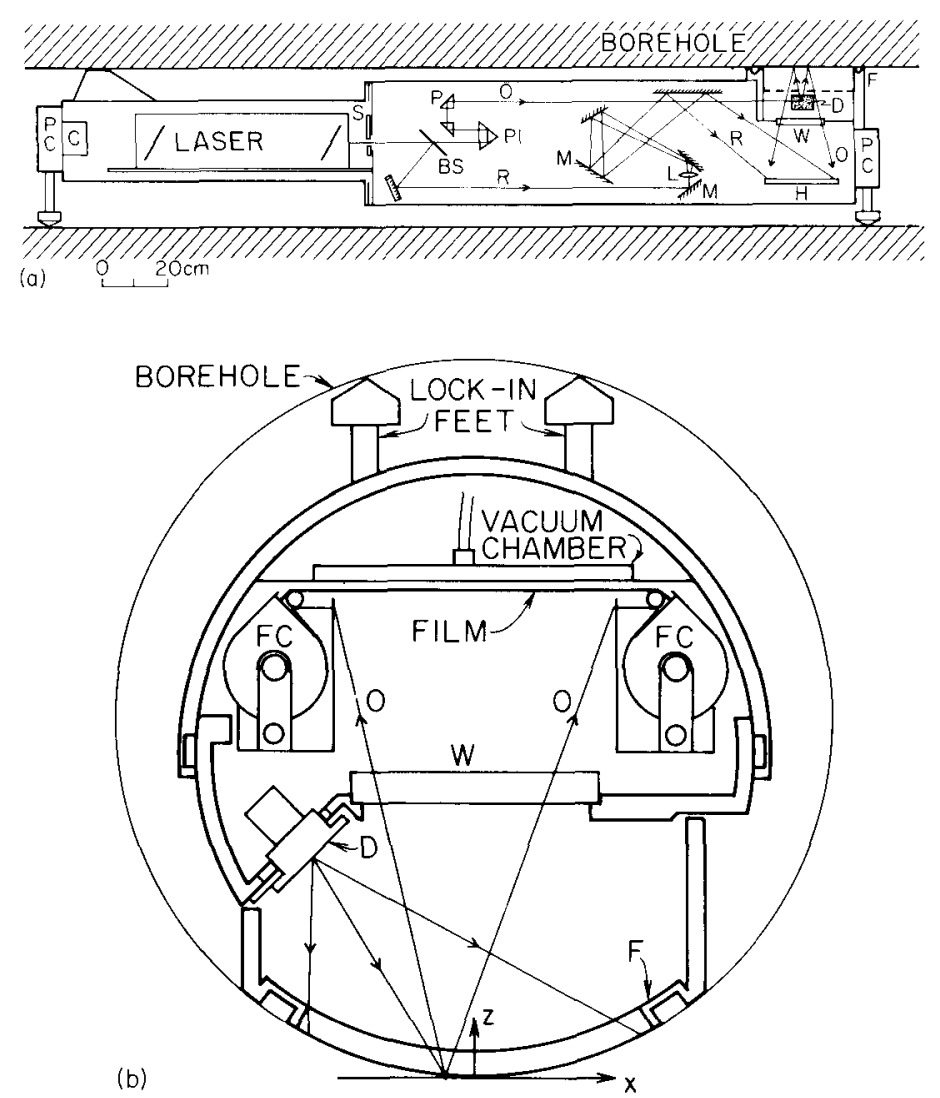

Figure 5. (a) Cross-section of the optics module of the holographic in situ stressmeter. Length scale is shown; the diameter is $29 \mathrm{~cm}$ and is slightly exaggerated for clarity. (b) Detailed top view of the holographic recording system and film transport mechanism. Abbreviations: S, shutter; P, P1, prisms; M, mirror; L, lens; R, reference beam; O, object beam; D, diffuser; $W$, window; $H$, holographic film plane; C, compass; PC, pneumatic cylinder; BS, beamsplitter; F, flange; FC, film cassette. 
the holographic film plane, $\mathrm{H}$, by a series of mirrors. Three prisms are used to steer the object beam on to a ground-glass diffuser and evenly illuminate the object at an incidence angle of about $45^{\circ}$ (Fig. 5b). The position of prism P1 (Fig. 5a) is adjustable so that the path lengths of the object and reference beams from $B S$ to $H$ are equal to within the coherence length of the laser ( $\sim 20 \mathrm{~cm}$ ), a necessary condition to produce bright holograms with good contrast. Light reflected by the object enters the camera through a window and exposes the film (Fig. 5b). It is noteworthy that no lenses are used to focus or image the object in any way.

A polyester-based holographic film, rather than glass plates, is used to record the holograms so that several measurements can be made without bringing the instrument to the surface. We have found that Agfa 10 E75 holographic film provides a satisfactory balance between resolution and speed, and has been used in most of our work. The film is loaded on two 70-mm cassettes (FC, Fig. 5b) and is remotely advanced over the film plane by a transport mechanism designed by Cohn (1983). During each exposure (about $2 \mathrm{~s}$ for a single exposure hologram) and through the course of a double exposure experiment the film must remain flat against the film plane. A partial vacuum is created in a chamber and acts on the film through a series of perforations in the film plane, thus fixing the film in place.

The entire optical apparatus is housed within a $3.2-\mathrm{mm}$ thick aluminium shell, which is water tight and may be pressurized during operation. A compass at the bottom of the laser compartment indicates the orientation of the instrument to the operator on the surface. When the module is at a desired depth and orientation, the unit is firmly secured in place by extending three lock-in feet; two at the camera end and one at the laser end. The lock-in feet are driven by pneumatic cylinders (PC) operated at a pressure of $0.7 \mathrm{MPa}$. Nylon support lines attached to the side of the control module connect it to the drill module, from which it is suspended without any rigid connection.

\subsection{DRILL MODULE}

The drill module, shown in Fig. 6, consists of two sections: a drill which cuts the stressrelieving hole, and a control section containing all of the valves and electrical connections required to actuate the various pneumatic and electrical components of the stressmeter. The drill is adjacent to the holographic camera and is exposed to the borehole environment, whereas the control section is enclosed in a water tight aluminium shell and connected to the electrical and high-pressure gas supply lines (umbilical), which run to the surface. A tungsten-carbide tipped masonry drill bit is screwed on to the end of a stainless steel arm attached to the drill casing. The drill casing contains an electric motor and pneumatic cylinder used to drive and extend the drill, respectively.

The control section contains the hardware used to direct power and high-pressure gas to each device on the stressmeter, so that a given function may be activated or stopped. Each of the pneumatically powered functions (e.g. module lock-in) is controlled by a simple logical circuit consisting of a pair of three-way magnetic solenoid valves (Minimatic EVO-3).

The entire sequence of operations that comprise a single measurement are as follows. the stressmeter is oriented in the desired position, securely locked in place with the pneumatically driven feet, and a fresh section of film is advanced on to the film plane. After an exposure of the undisturbed borehole wall is taken, the drill arm is extended by a pneumatic cylinder so that the drill bit is approximately centred within the field of view of the holographic camera, and the drilling operation commences. During drilling the entire drill housing is advanced over four steel rods and driven towards the borehole wall by a pair of pneumatic flat jacks at a rate of $\sim 30 \mathrm{~mm} \mathrm{~min}^{-1}$. Magnetic reed switches indicate when the 


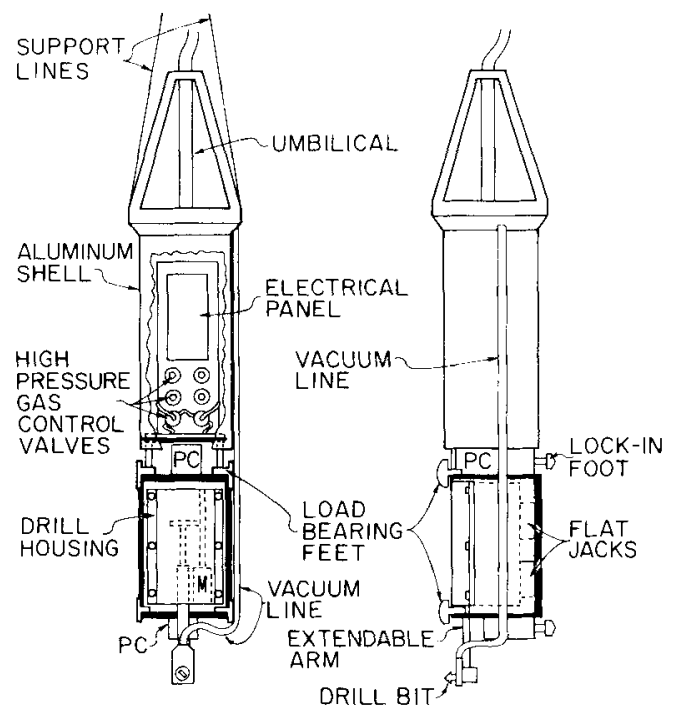

Figure 6. Front view (at left), and side view (at right) of the drill module. An interior view of the control section is shown on the left illustration.

drill is either fully advanced or retracted. After the hole is completed the drill is retracted and another exposure of the borehole wall surface is made, thus completing the doubleexposure interferogram.

It is extremely important that the drill and optics modules remain separated while the drill is being operated. If any strong mechanical vibrations from drilling are transmitted to the optics module, the position of the optics module will be disturbed. Relative motions between the borehole and optics module of greater than several micrometres in between exposures will obscure the stress relief fringes and result in an unintelligible hologram. Nylon support lines joining the modules are effective in damping high-frequency drill noise, providing no parts of the modules are in contact. To check that the modules are separated, the electrical resistance between them is continuously monitored, and any contact is easily detected before a stress measurement is attempted. In the event that the modules are in contact, they are unlocked and repositioned.

Remote control of the stressmeter from the surface is achieved by a control panel. The panel is connected to a $110 \mathrm{~V}$ line and also supplies power to the instrument. In addition to controlling instrument functions, the control panel has indicator lights to show the position of all instruments (i.e. lock-in feet extended or retracted, drill head extended or retracted, etc.), gauges for monitoring the electrical resistance between the two modules and power consumption of the drill, and an indicator to show the orientation of the stressmeter within the borehole.

\section{Analysis of the data}

In Plate 1 we have shown a photograph of a typical interference hologram, consisting of the object image and a series of dark fringes. Each dark and light fringe on the reconstructed image corresponds to a contour of constant phase shift between the first and second exposures of the interferogram, as described in the previous section. Interpretation of the interferogram requires a relationship between displacement of the object and phase shift of 


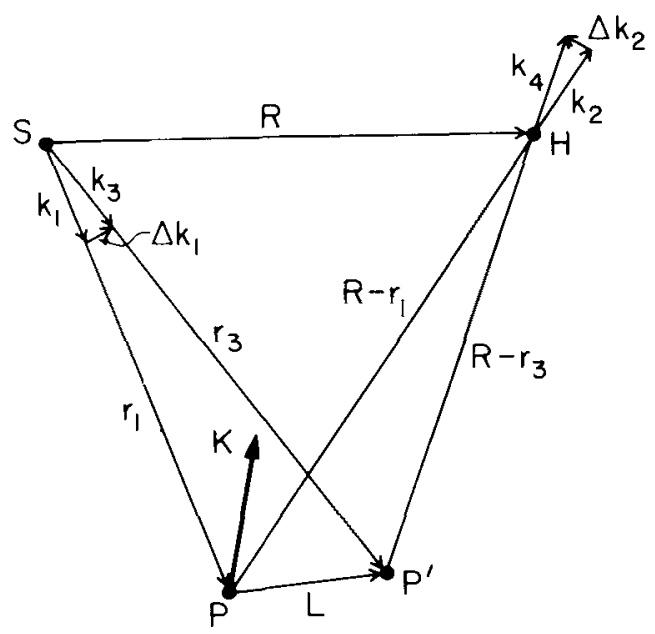

Figure 7. Vector diagram for defining the interference conditions on double exposure holograms. Point $\mathbf{P}$ on the object reflects light from source $S$ to $H$ on the film plane. After a deformation, $P$ is displaced to $P^{\prime}$.

light reflected to the holographic film. Once the displacements on the object surface have been determined, it is a separate problem to relate this deformation to a stress state.

Our method of interpreting the interferograms follows the formulation of Sollid (1969a, b). Consider point $P$ on an object that reflects light from a point source illuminator $S$ to a given observation point on the holographic film plane $\mathrm{H}$ (Fig. 7). Wavevectors $\mathbf{k}_{1}$ and $\mathbf{k}_{2}$ correspond to light striking and reflected from $\mathrm{P}$, respectively. A holographic exposure is taken and $\mathbf{P}$ is then displaced to $\mathbf{P}^{\prime}$, with $\mathbf{L}$ being the vector displacement. Another exposure is taken. The phase of light reaching $\mathrm{H}$ from $\mathrm{P}$ during each exposure is:

$\phi=\mathbf{k}_{1} \cdot \mathbf{r}_{1}+\mathbf{k}_{2} \cdot\left(\mathbf{R}-\mathbf{r}_{1}\right)+\phi_{\mathrm{r}}$

$\phi^{\prime}=\mathbf{k}_{3} \cdot \mathbf{r}_{3}+\mathbf{k}_{4} \cdot\left(\mathbf{R}-\mathbf{r}_{3}\right)+\phi_{\mathrm{r}}$

where $r_{1}$ and $r_{3}$ are position vectors from $S$ to the point in the original and deformed states, respectively, and $\phi_{\mathrm{r}}$ is an arbitrary phase shift due to reflection from the surface. The phase change of light resulting from the displacement, $\delta$, is simply:

$\delta=\phi^{\prime}-\phi$.

Let $\Delta \mathbf{k}_{1}=\mathbf{k}_{3}-\mathbf{k}_{1}$ and $\Delta \mathbf{k}_{2}=\mathbf{k}_{4}-\mathrm{k}_{2}$. From equations $(4-6)$ we then obtain:

$\delta=\left(\mathbf{k}_{2}-\mathbf{k}_{1}\right) \cdot\left(\mathbf{r}_{1}-\mathbf{r}_{3}\right)+\Delta \mathbf{k}_{1} \cdot \mathbf{r}_{3}+\Delta \mathbf{k}_{2} \cdot\left(\mathbf{R}-\mathbf{r}_{3}\right)$.

Because the deformations are small the magnitude of $\mathbf{L}$ is much less than $\mathbf{r}_{1}$ or $\mathbf{r}_{3}$, so to a very good approximation $\Delta \mathbf{k}_{1}$ is normal to $\mathbf{r}_{3}$ and $\Delta \mathbf{k}_{2}$ is normal to $\left(\mathbf{R}-\mathbf{r}_{3}\right)$. Denoting $\left(\mathbf{k}_{\mathbf{2}}-\mathbf{k}_{\mathbf{1}}\right)$ as $\mathbf{K}$, the sensitivity vector (Vest 1979), equation (7) is simplified to:

$\delta \approx\left(\mathbf{k}_{2}-\mathbf{k}_{1}\right) \cdot \mathbf{L}=\mathbf{K} \cdot \mathbf{L}$.

The vector $\mathbf{K}$ is a function only of the geometry of the holographic apparatus and the wavelength of laser light used. At each point on the interferogram where a dark fringe is observed we know that:

$\delta=(2 N+1) \pi \quad$ (dark fringe $)$

$\delta=2 N \pi \quad$ (bright fringe) 
where $N$ is an integer. The phase change $\delta$ may easily be estimated to within half of a fringe. Therefore, the only unknown in equation ( 8 ) is the displacement, $\mathbf{L}$, which may be solved for. Note from Fig. 7 that the vector $\mathbf{k}_{2}$ varies with the position of the observer. The fringe pattern therefore seems to move about as one views the interferogram from different positions. By using widely separated portions of the hologram for photographing the image, different fringe patterns will be obtained, and this property of holographic interferometry can be used to constrain the individual components of the vector L (Dhir \& Sikora 1972), assuming that the zero-order fringe in each pattern is identifiable. Even if a zero-order fringe cannot be identified the displacement field may still be determined by measuring the differences in phase shift, $\delta$, obtained by viewing a given point on the object from several different directions (Aleksandrov \& Bonc-Bruevich 1967). In this case one measures the number of fringes which cross a point on the holographic image as the observation point on the film plane changes.

From equation (8) it is seen that holographic interferometry records only the projection of the displacement vector on the sensitivity vector $\mathbf{K}$. Thus, the process is very sensitive to displacements parallel to $\mathbf{K}$ and insensitive to motions normal to $\mathbf{K}$. The optical geometry of our holographic camera (Fig. 5) is therefore most sensitive to object deformations toward or away from the film plane and least sensitive to motion parallel to the long axis of the stressmeter or borehole.

An intrinsic ambiguity of the holographic interferometry technique arises with regard to the direction of the displacement. Either a positive or negative sense of displacement will yield an identical phase change of light and produce identical fringe patterns. Equivalently, the sign of the integer $N$ (equation 9) may be either positive or negative, and the resulting interferograms will be indistinguishable. One must use additional information on the physical basis for the deformation to choose between the two possibilities.

Equations (8) and (9) form the general basis for interpreting holographic interferograms. However, the data analysis procedure itself will vary depending upon the details of the experiment and data base. We have adopted a direct forward modelling approach, which is to assume an initial set of displacements on a collection of grid points on the object. The fringe pattern corresponding to the displacement field is calculated by equations ( 8 ) and ( 9 ) and then compared to the fringe pattern observed on the interferogram. The displacements are then perturbed to improve agreement between the two fringe patterns, and this procedure is repeated until a satisfactory match between the theoretical and observed fringe patterns is obtained. Although the forward modelling approach is somewhat subjective, it has the advantage of being computationally simple. We anticipate that more sophisticated methods of treating the data, such as a formal inversion of the fringe pattern to obtain the displacement field (i.e. Dhir \& Sikora 1972), will parallel improvements in the stressmeter hardware.

\section{Concentration of far-field stresses by the borehole}

The concentration of far-field stresses at a borehole has been well studied (Hiramatsu \& Oka 1962; Fairhurst 1964; Leeman \& Hayes 1966), and the theoretical aspects of determining far-field stresses by holographic interferometry closely parallel the overcoring-strain gauge work of Leeman \& Hayes (1966), and the stress relief method of Bock, Foruria \& Lequerica (1984). These methods require three measurements at the borehole wall surface to determine far-field stresses at a given depth in the borehole.

Consider an infinite rock mass under a uniform state of stress, into which a borehole is drilled. We define an orthogonal coordinate system $x, y, z$ (Fig. 8), such that the $z$-axis is 


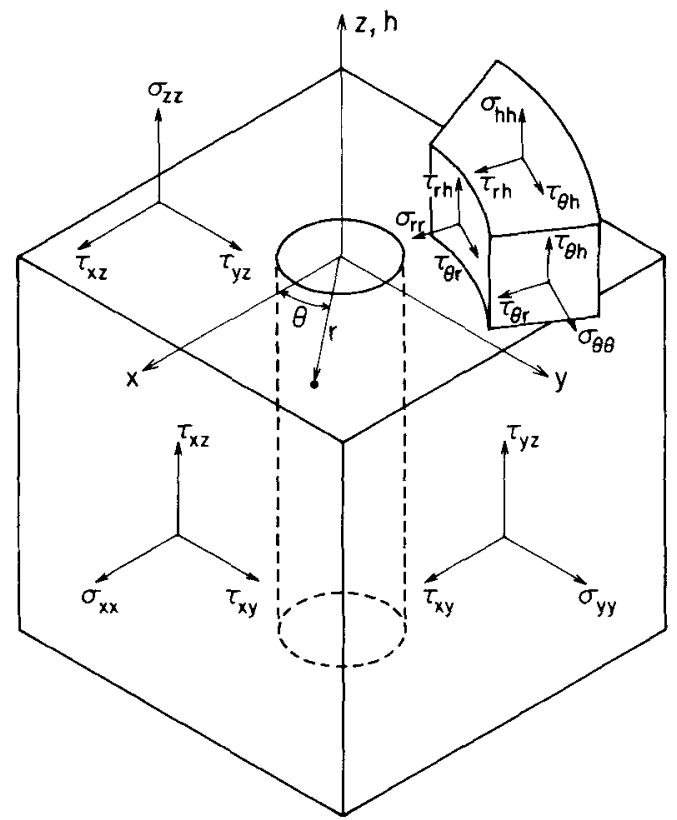

Figure 8. Definitions of coordinate systems. A Cartesian system $(x, y, z)$ is used to describe the far-field stress components $\left(\sigma_{x x}, \sigma_{y y}, \sigma_{z z}, \tau_{x y}, \tau_{x z}, \tau_{y z}\right)$, and is defined such that the $z$ axis coincides with the long axis of the borehole. A cylindrical system $(r, \theta, h)$ is primarily used to describe the stress state at the borehole surface. The $z$-and $h$ axes coincide; $\theta=0$ along the $x$-direction. A positive value of $\theta$ is shown. Sign convention for stresses is: compressive normal stress is negative, and a positive sense of shear is as shown in the figure.

coincident with the borehole axis. The uniform far-field stresses are given by $\sigma_{x x}, \sigma_{y y}, \sigma_{z z}$, $\tau_{x y}, \tau_{x z}, \tau_{y z}$, where $\sigma$ is a normal, or on-diagonal stress component, and $\tau$ is a shear, or offdiagonal stress component. The stress sign convention followed is that of Timoshenko \& Goodier (1970), i.e. compressional normal stresses are negative and tensional normal stresses are positive. All shear stresses shown in Fig. 8 are of positive sign. We further define a cylindrical coordinate system $r, \theta, h$ (Fig. 8) such that the $h$-axis is also coincident with the axis of the borehole. The stress state around the borehole is described in terms of $r, \theta, h$ as $\sigma_{r r}, \sigma_{\theta \theta}, \sigma_{h h}, \tau_{r \theta}, \tau_{r h}, \tau_{\theta h}$. At the borehole wall ( $r=1$ borehole radius), the only nonvanishing stresses are given by (Leeman $\&$ Hayes 1966):

$\sigma_{\theta \theta}(\theta)=\sigma_{x x}+\sigma_{y y}-2\left(\sigma_{x x}-\sigma_{y y}\right) \cos 2 \theta-4 \tau_{x y} \sin 2 \theta$

$\sigma_{h h}(\theta)=-\nu\left[2\left(\sigma_{x x}-\sigma_{y y}\right) \cos 2 \theta+4 \tau_{x y} \sin 2 \theta\right]+\sigma_{z z}$

$\tau_{\theta h}(\theta)=2\left(-\tau_{x z} \sin \theta+\tau_{y z} \cos \theta\right)$

where $\nu$ is Poisson's ratio. Thus, at a given $z$, finding $\sigma_{\theta \theta}(\theta), \sigma_{h h}(\theta)$, and $\tau_{\theta h}(\theta)$ for three different azimuths, $\theta_{1}, \theta_{2}$, and $\theta_{3}$ around the borehole, allows us to solve for the far-field stresses. For example, if one chooses $\theta_{1}=0, \theta_{2}=\pi / 4, \theta_{3}=\pi / 2$, the far-field stresses will be given by:

$\sigma_{x x}=\frac{1}{8}\left(3 \sigma_{\theta \theta}(\pi / 2)+\sigma_{\theta \theta}(0)\right)$ 
$\sigma_{y y}=\frac{1}{8}\left[3 \sigma_{\theta \theta}(0)+\sigma_{\theta \theta}(\pi / 2)\right]$

$\sigma_{z z}=\sigma_{h h}(0)+\frac{\nu}{2}\left[\sigma_{\theta \theta}(\pi / 2)-\sigma_{\theta \theta}(0)\right]$

$\tau_{x y}=\frac{1}{8}\left[\sigma_{\theta \theta}(0)+\sigma_{\theta \theta}(\pi / 2)-2 \sigma_{\theta \theta}(\pi / 4)\right]$

$\tau_{y z}=\frac{1}{2} \tau_{\theta h(0)}$

$\tau_{x z}=-\frac{1}{2} \tau_{\theta h}(\pi / 2)$

The problem therefore reduces to finding the six borehole wall stresses: $\sigma_{\theta}(0), \sigma_{\theta \theta}(\pi / 4)$, $\sigma_{\theta \theta}(\pi / 2), \sigma_{h h}(0), \tau_{\theta h}(0), \tau_{\theta h}(\pi / 2)$. Ascertaining the remaining three borehole stresses: $\tau_{\theta h}(\pi / 4), \sigma_{h h}(\pi / 4)$, and $\sigma_{h h}(\pi / 2)$, serves as a check on the internal consistency of the calculations.

The stressmeter records a displacement field during the double exposure hologram. A model of the displacements that result from drilling a stress-relief hole between exposures is thus required to deduce the stress state. The model we have chosen is the plane stress case of a hole in an infinite isotropic elastic plate of thickness $t$. The appropriateness of this model rests on the following assumptions:

(1) The borehole wall can be approximated by a flat plate over the viewing area of the hologram.

(2) The borehole wall stresses do not change rapidly over the viewing area, or into the borehole wall over the depth of the stress-relief hole.

Fig. 9 illustrates the variation of azimuthal stress, $\sigma_{\theta \theta}$ (normalized to the maximum far-field stress), at the borehole wall surface ( $r=D=1$ borehole radius) for several simple states of far-field stress. This figure also shows the difference in $\sigma_{\theta \theta}$ over the depth, $\Delta$, of a typical stress-relief hole $(r=D+\Delta \approx 1.1 D)$. In the limiting case of uniaxial stress, it is clear that $\sigma_{\theta \theta}$ can change by as much as 60 per cent of the peak-to-peak stress over the viewing area of the hologram (shown by segment A-B). At the other extreme of a biaxial stress with $\sigma_{x x}=\sigma_{y y}$ (Fig. 9), the stress $\sigma_{\theta \theta}$ is uniform at all azimuths around the borehole surface. Obviously, the situation within any borehole will fall somewhere between these two cases. Stress measurements at depth in mines (McGarr \& Gay 1978) and in boreholes by hydrofracture (Zoback, Tsukahara \& Hickman 1980; Bredehoeft et al. 1976; Haimson 1977) demonstrate that the horizontal principal stresses usually do not differ by more than a factor of 2 , suggesting that the change in $\sigma_{\theta \theta}$ with $\theta$ is not as extreme as for the uniaxial case. In Fig. 9 , the borehole wall stress $\sigma_{\theta \theta}$ is plotted for a principal stress state of $\sigma_{\min }=\sigma_{\max } / 2$. The curve for this case does not change as rapidly as the uniaxial case, although changes over the viewing area of the hologram are still significant. One way to reduce errors resulting from the change of $\sigma_{\theta \theta}$ around the borehole wall is to confine our analysis of the holographic data to a small region surrounding the side-core hole.

In Fig. 9 it is also demonstrated that $\sigma_{\theta \theta}$ changes rapidly as $r$ increases. The stress-relief holes in our field tests drilled to a depth of $\Delta=1.5 \mathrm{~cm}(=0.1 D)$, and the stress $\sigma_{\theta \theta}$ at this particular depth is plotted. $\sigma_{\theta \theta}$ decreases in magnitude with increasing distance from the 


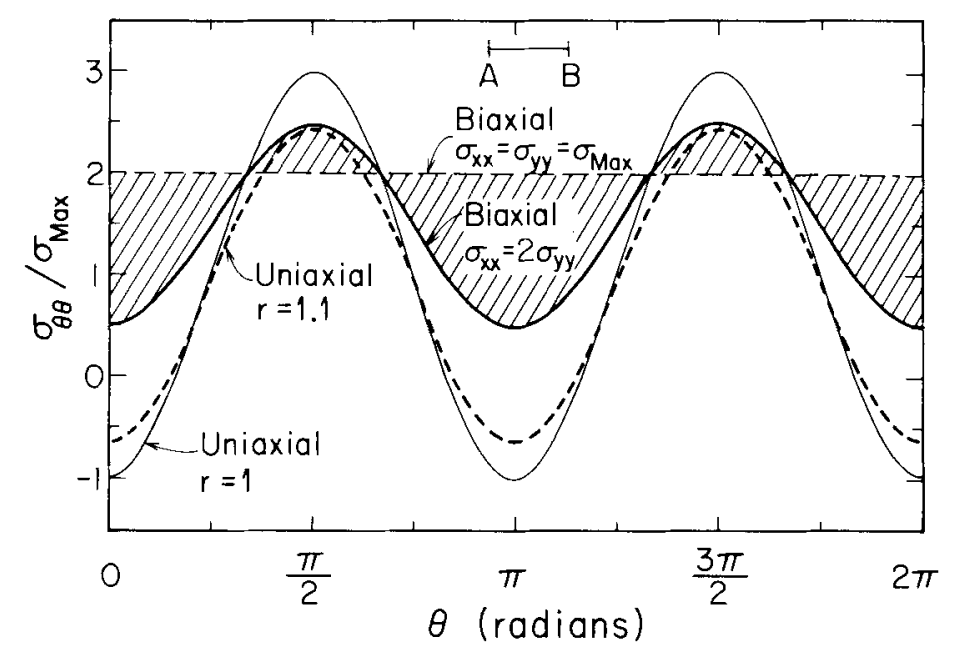

Figure 9. Azimuthal variation in $\sigma_{\theta \theta}$ (hoop stress around borehole) normalized to the maximum principal far field stress, $\sigma_{\max }$. Radius $r$ is in units of borehole radii. The light solid line and heavy dashed line represent $\sigma_{\theta \theta}$ at the borehole wall $(r=1)$ and at the depth of a typical stress-relief hole $(r=1.1)$, respectively, for a uniaxial far-field stress state $\sigma_{x x}=\sigma_{\max }$. The light dashed line shows a constant $\sigma_{\theta \theta}$ for a biaxial stress state, $\sigma_{x x}=\sigma_{y y}=\sigma_{\max }$. The heavy solid line shows $\sigma_{\theta \theta}$ for a stress state of $\sigma_{x x}=2 \sigma_{y y}=$ $\sigma_{\max }$. Previous work (see text) indicates that the in situ state of stress usually lies in the hatched region between the light dashed line and the heavy solid line. Segment AB is the angular view of the borehole wall recorded on the hologram.

borehole wall; $\sigma_{\theta \theta}$ at the depth of the stress-relief hole can differ from the borehole wall stress by as much as 40 per cent. Thus, the magnitude of $\sigma_{\theta \theta}$ measured at the borehole wall may be an average of the stresses encountered along the depth of the side-core hole. Although these errors may be reduced by drilling a shallower stress-relief hole, this practice would worsen our approximation of the stress-relief hole as a throughgoing hole in a stressed plate.

From the above discussion, we conclude that the hole in a plate model of stress relief has severe limitations. However, it is adequate for our initial calculations, providing semiquantitative measures of the displacement field induced by the drilling of a stress-relief hole. This simple model should, in fact, be a good approximation in the near vicinity of the stressrelief hole.

Fig. 10 shows the position of a stress-relief hole at azimuth $\theta$ within the borehole. With the plate model discussed above, a section of the borehole wall and adjacent rock mass (shown with a heavy outline in Fig. 10) is approximated as a flat plate tangent to the borehole wall. In order to calculate the displacements induced on the surface of the model plate by drilling a stress-relief hole, we define a cylindrical coordinate system $(\rho, \phi, \zeta)$, as shown in Fig. 11. The $\zeta$-axis is along the axis of the stress-relief hole, and the origin of the coordinate system is on the tangent to the borehole surface. Also shown in Fig. 11 are the stresses $\sigma_{\theta \theta}$, $\sigma_{h h}$, and $\tau_{\theta h}$ on the borehole surface, which we use as the stresses exerted on the flat plate at infinity. Because all other stresses are zero at the borehole wall, we further assume that a state of plane stress exists within the model plate. Note that it is a separate issue to relate $\sigma_{\theta \theta}, \sigma_{h h}$, and $\tau_{\theta h}$ to the far-field stresses (equations 10-12).

We require a solution for the displacements, $U$, of the borehole wall surface due to the 


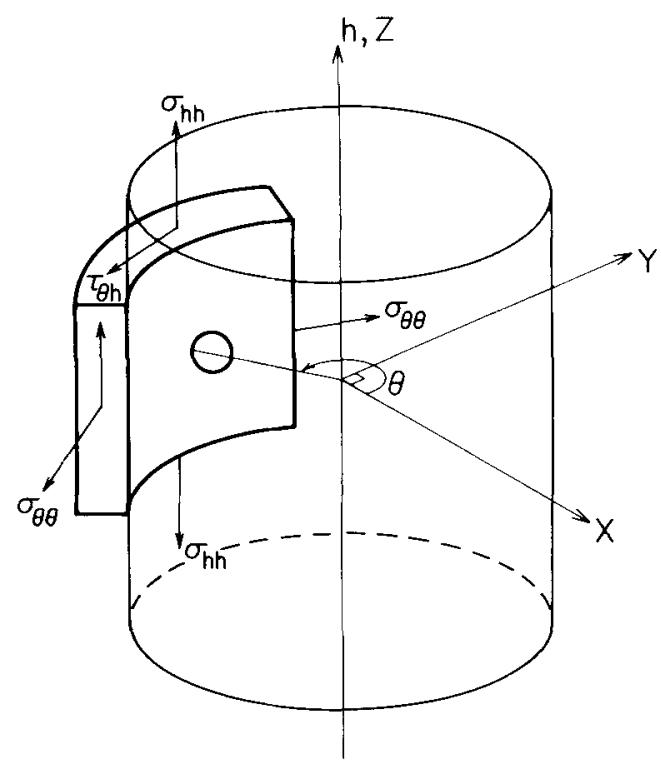

Figure 10. Stress relief hole located at azimuth $\theta$ in a borehole. In our data analysis, the rock mass adjacent to the stress-relief hole (heavy outline), is approximated by a flat plate, with stsesses $\sigma_{\theta} \theta, \sigma_{h h}$, $\tau_{\theta h}$, acting on the plate at infinity.

drilling of the stress-relief hole. The solution for the stress concentration for a hole in a plate subjected to plane stress is well known (Timoshenko \& Goodier 1970; Obert \& Duvall 1967). Referring to the plate shown in Fig. $11, \sigma_{\phi \phi}, \sigma_{\rho \rho}$ and $\tau_{\phi \rho}$ are finite, whereas $\sigma_{\zeta \zeta}$, $\tau_{\zeta \phi}$ and $\tau_{\zeta \rho}$ vanish. The stress-displacement relations for plane stress in a cylindrical

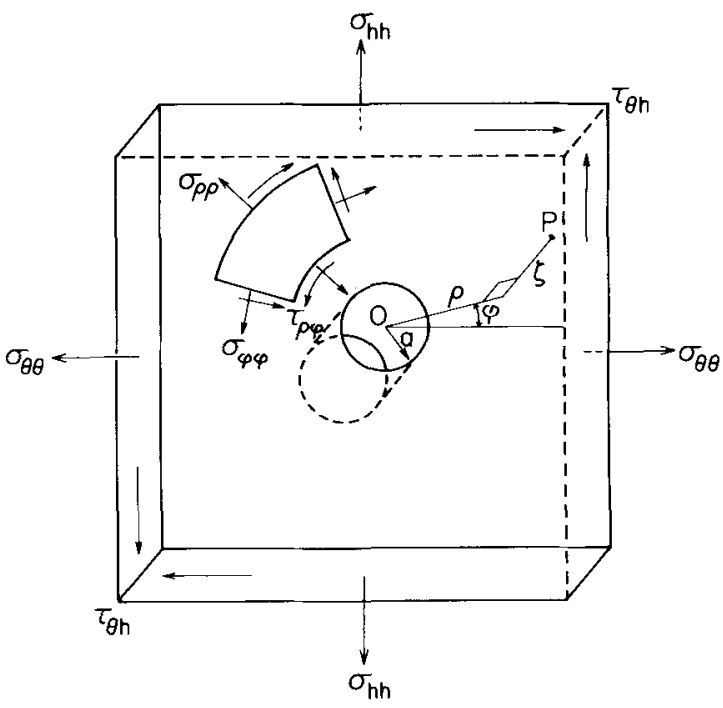

Figure 11. A plate with a throughgoing hole under plane stress. We model the near face of the plate as the borehole surface and stress relief hole (see Fig. 10). Point $P$ on the plate is specified by coordinates $(\rho, \phi, \xi)$, which are defined in terms of the natural cylindrical coordinate system of the stress-relief hole. 
coordinate system are:

$$
\begin{aligned}
& \frac{\partial U_{\rho}}{\partial \rho}=\frac{1}{E}\left(\sigma_{\rho \rho}-\nu \sigma_{\phi \phi}\right) \\
& \frac{U_{\rho}}{\rho}+\frac{1}{\rho} \frac{\partial U_{\phi}}{\partial \phi}=\frac{1}{E}\left(\sigma_{\phi \phi}-\nu \sigma_{\rho \rho}\right) \\
& \frac{1}{\rho} \frac{\partial U_{\rho}}{\partial \phi}+\frac{\partial U_{\phi}}{\partial \rho}-\frac{U_{\phi}}{\rho}=\frac{2(1+\nu)}{E} \tau_{\rho \phi} \\
& \frac{\partial U_{\zeta}}{\partial \zeta}=-\frac{\nu}{E}\left(\sigma_{\rho \rho}+\sigma_{\phi \phi}\right) .
\end{aligned}
$$

Denoting the principal stresses on the plate at infinity by $S_{x}$ and $S_{y}$, the stresses $\sigma_{\rho \rho}, \sigma_{\phi \phi}$, $\tau_{\rho \phi}$ are given by

$$
\begin{aligned}
& \sigma_{\rho \rho}=\frac{1}{2}\left(S_{x}+S_{y}\right)\left(1-\frac{a^{2}}{\rho^{2}}\right)+\frac{1}{2}\left(S_{x}-S_{y}\right)\left(1+\frac{3 a^{4}}{\rho^{4}}-\frac{4 a^{2}}{\rho^{2}}\right) \cos 2 \phi \\
& \sigma_{\phi \phi}=\frac{1}{2}\left(S_{x}+S_{y}\right)\left(1+\frac{a^{2}}{\rho^{2}}\right)-\frac{1}{2}\left(S_{x}-S_{y}\right)\left(1+\frac{3 a^{4}}{\rho^{4}}\right) \cos 2 \phi \\
& \tau_{\rho \phi}=-\frac{1}{2}\left(S_{x}-S_{y}\right)\left(1-\frac{3 a^{4}}{\rho^{4}}+\frac{2 a^{2}}{\rho^{2}}\right) \sin 2 \phi
\end{aligned}
$$

where $\phi$ is measured from the direction of $S_{x}$ and $a$ is the hole radius. Using the above sets of equations (19-25), the displacements $U_{\rho}, U_{\phi}, U_{\zeta}$ for a plate with a hole at the origin, subjected to principal stresses $S_{x}$ and $S_{y}$ are:

$$
\begin{aligned}
U_{\rho}= & \frac{1}{2 E}\left[\left(S_{x}+S_{y}\right)\left(\rho+\frac{a^{2}}{\rho}\right)+\left(S_{x}-S_{y}\right)\left(\rho-\frac{a^{4}}{\rho^{3}}+4 \frac{a^{2}}{\rho}\right) \cos 2 \phi\right] \\
& -\frac{\nu}{2 E}\left[\left(S_{x}+S_{y}\right)\left(\rho-\frac{a^{2}}{\rho}\right)-\left(S_{x}-S_{y}\right)\left(\rho-\frac{a^{4}}{\rho^{3}}\right) \cos 2 \phi\right] \\
U_{\phi}= & \frac{1}{2 E}\left[-\left(S_{x}-S_{y}\right)\left(\rho+\frac{2 a^{2}}{\rho}+\frac{a^{4}}{\rho^{3}}\right) \sin 2 \phi\right] \\
& -\frac{\nu}{2 E}\left[\left(S_{x}-S_{y}\right)\left(\rho-2 a^{2} / \rho+a^{4} / \rho^{3}\right) \sin 2 \phi\right] \\
U_{\zeta}= & -\frac{\nu}{E}\left[\left(S_{x}+S_{y}\right)-\left(S_{x}-S_{y}\right) \frac{2 a^{2}}{\rho^{2}} \cos 2 \phi\right] \frac{t}{2}
\end{aligned}
$$

where $t$ is the thickness of the model plate. We again emphasize that the above displacements apply to a plate with an existing hole, subjected to stresses at infinity. To approximate the borehole, however, the model we require is a pre-stressed plate into which a hole is drilled. By the principle of superposition the desired state is equivalent to the displacements on a plate with a pre-existing hole subjected to stress at infinity, less the displacements on a plate 
without a hole subjected to stress at infinity. The latter case is given by:

$$
\begin{aligned}
& U_{\rho}=\frac{1}{E}\left[\left(S_{y}-\nu S_{x}\right) \rho \sin ^{2} \phi+\left(S_{x}-\nu S_{y}\right) \rho \cos ^{2} \phi\right] \\
& U_{\phi}=\frac{1}{E}\left[(1+\nu)\left(S_{y}-S_{x}\right) \rho \sin \phi \cos \phi\right] \\
& U_{\zeta}=-\frac{\nu}{E}\left(S_{x}+S_{y}\right) t / 2 .
\end{aligned}
$$

Thus, in terms of the borehole surface stresses the displacements due to drilling a hole in a pre-stressed plate are:

$$
\begin{aligned}
U_{\rho}= & \frac{1}{2 E}\left\{\left(\sigma_{\theta \theta}+\sigma_{h h}\right)(1+\nu) \frac{a^{2}}{\rho}+\left(\sigma_{\theta \theta}-\sigma_{h h}\right)\left[4 \frac{a^{2}}{\rho}-(1+\nu) \frac{a^{4}}{\rho^{3}}\right] \cos 2 \phi\right. \\
& \left.+2 \tau_{\theta z}\left[4 \frac{a^{2}}{\rho}-(1+\nu) \frac{a^{4}}{\rho^{3}}\right] \sin 2 \phi\right\} \\
U_{\phi}= & -\frac{1}{2 E}\left\{(1-\nu) \frac{2 a^{2}}{\rho}+(1+\nu) \frac{a^{4}}{\rho^{3}}\left[\left(\sigma_{\theta \theta}-\theta_{h h}\right) \sin 2 \phi-2 \tau_{\theta z} \cos 2 \phi\right]\right\} \\
U_{\zeta}= & \frac{\nu a^{2} t}{E \rho^{2}}\left[\left(\sigma_{\theta \theta}-\sigma_{h h}\right) \cos 2 \phi+2 \tau_{\theta h} \sin 2 \phi\right] .
\end{aligned}
$$

Equations $(32-34)$ are used to determine the displacements due to a given set of stresses in the forward modelling of the observed fringe patterns. An uncertainty in the use of (34) for determining $U_{\zeta}$ is the plate thickness, $t$. For a thick plate under plane stress, the displacement $U_{\zeta}$ increases linearly from a minimum value of $U_{\zeta}=0$ on the plane midway between the plate surfaces, to a maximum value at the plate surface, and as the thickness of the plate increases so does the magnitude of $U_{\zeta}$. However, for a finite depth hole it is not clear as to what 'plate thickness' should be adopted to describe the displacements $U_{\zeta}$ normal to the plate surface. In our data analysis we have assumed a plate thickness equal to twice the depth of the stress-relief hole. This is equivalent to assuming that the $\zeta$ displacements in the rock mass are small at the depth of the stress-relief hole $(r=D+\Delta)$ thus mimicking the symmetry plane of a plate with thickness $t=2 \Delta$. Inasmuch as the present camera geometry is most sensitive to motions toward and away from the holographic film plane, an important improvement to be made in our data analysis is to determine a justifiable model of the normal displacements, $U_{\xi}$. However, it should also be recognized that at the borehole surface, the calculated normal displacements amount to only about 25 per cent of the inplane displacements, which are modelled fairly accurately in the neighbourhood of the stress-relief hole by the simple plate theory.

\section{Results}

\subsection{LABORATORY TESTS}

Two types of laboratory tests have been conducted with the holographic stressmeter. The first set of tests consisted of double exposure interferograms of metal plates or bars which were displaced a known amount in between the two holographic exposures. The purpose 
of these tests was twofold: to ensure that the measured geometry of the holographic camera was correct and to demonstrate that the interference fringe pattern resulting from a known displacement can be modelled accurately. In each calibration interferogram the test object was rotated about an axis parallel to either the $x, y$, or $z$-axes, as defined in Fig. 8 , and the displacement at some point on the object was measured within \pm 15 per cent by a mechanical dial gauge. Because the test objects were rotated rather than uniformly translated, the object displacement gradually increased from a value of zero at the hinge point. Hence, an order number could be unambiguously assigned to each fringe on the resulting interferograms. This simplified interpretation of the observed fringe patterns and allowed a direct comparison with the theoretical patterns, as shown in Plate 2. In this figure, a lever (no. 1) is rotated about a hinge parallel to the $z$-axis by means of turning a screw. Motion is transmitted to the other levers, but the motion in each successive lever is reduced due to the position of the hinge points. The motion reduction is evident from the contrasting fringe density in levers 2 and 3 . The displacement of lever 3 was measured at point $\mathrm{P}$ as $7.6 \pm 1.2 \mu \mathrm{m}$, whereas the calculated displacement at $\mathrm{P}$ is $8.7 \mu \mathrm{m}$. Numerous tests of this kind, for different directions of displacement, were performed, and in all cases both the number and orientation of the observed fringes could be matched by displacements that were within the uncertainty of the measured values.

A second set of laboratory experiments, designed to simulate actual field conditions, was carried out in an artificial borehole. The artificial borehole into which the stressmeter is lowered consisted of a $30 \mathrm{~cm}$ internal diameter concrete pipe, $4 \mathrm{~m}$ in length. An apparatus for stressing samples is cemented into a window located midway down the length of the concrete tube. The stressing apparatus, and its configuration relative to the concrete artificial borehole, is shown in Fig. 12. A hydraulic jack, which exerts a uniaxial stress on the sample, is bolted to a stressing frame made of high tensile strength steel. Force is transmitted to the sample via a steel block to help achieve even contact and stress distribution over the side faces of the sample. The stressing apparatus is designed so that the frame and sample bear all of the load, and no stress is transmitted to the artificial borehole. By monitoring the fluid pressure on the hydraulic jack (Enerpac JMC 200), an approximately known stress state can

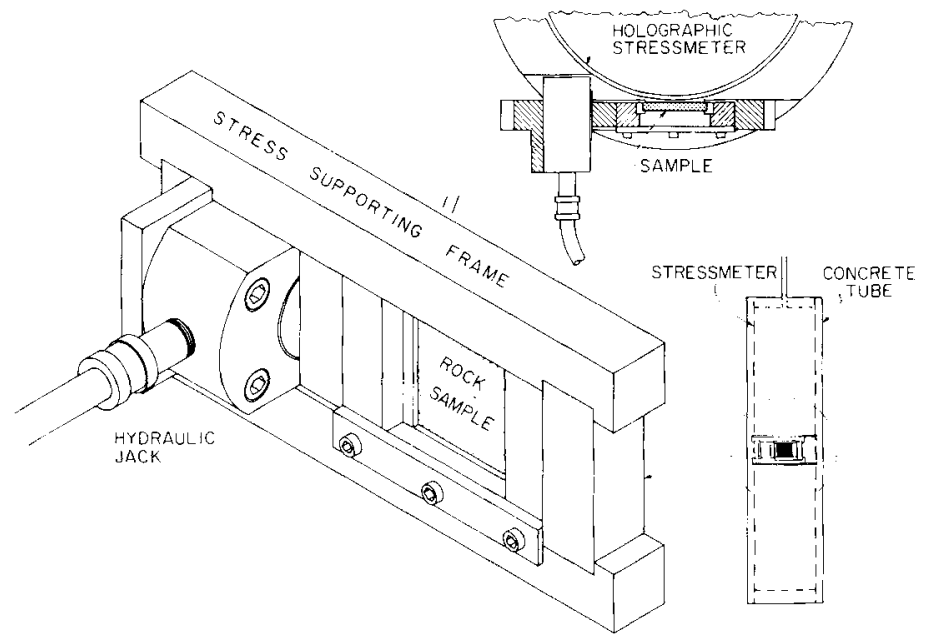

Figure 12. Apparatus used to stress samples uniaxially for our tests in an artificial borehole. The apparatus is shown at right cemented into a window in the artificial borehole. Top insert shows a cross-section of the stressing apparatus. 
be imposed upon a sample. When the stressmeter is operated in the concrete tube, the prestressed sample is drilled, thus allowing us to test the ability of the stressmeter to record strain-relief fringes in a simulated borehole environment before deploying it in the field.

Plate 3 shows the results of a test conducted in the artificial borehole. The sample is a $70 \times 70 \times 16 \mathrm{~mm}$ plate of $2024 \mathrm{~T}$ aluminium alloy subjected to a nominal uniaxial compressive stress of $120 \pm 20 \mathrm{MPa}$. For this test a $6.35 \mathrm{~mm}$ diameter bit was used with the stressmeter to drill a hole $6 \mathrm{~mm}$ deep into the pre-stressed plate. The sample was wrapped, around its periphery, with a strip of $0.05 \mathrm{~mm}$ thick teflon tape to assure even contact between the sample and stress transmitting parts and also to help minimize the presence of tractions between these components. In an independent set of tensile stress-strain measurements employing strain gauges, we obtained values of Young's modulus $E=75 \pm 4 \mathrm{GPa}$ and Poisson's ratio $\nu=0.345 \pm 0.017 \mathrm{GPa}$, in agreement with values given by Lyman (1971), and these results were used in our calculations. Plate 3 shows a theoretical fringe pattern for a test plate under an assumed horizontal uniaxial stress of $80 \mathrm{MPa}$. The theoretical fringe pattern in Plate 3 explains several features on the observed hologram; namely, the number and shape of both the circular, lobed fringes on the right (source) side of the stress relief hole, and the more gently curved, elongate fringes on the left side of the hole. The circular fringes close to the hole are primarily due to stress relief, whereas the elongate fringes are caused by a combination of a uniform sample translation in the $y$-direction, the boundary conditions for this specific type of laboratory test (see below), and, to a lesser degree, stress relief. A small translation of the stressmeter relative to the sample and stressing apparatus is clearly evidenced by the diagonal fringes on the steel parts adjacent to the sample (Plate 3 ).

There are, however, some discrepancies between the observed and calculated fringe pattern. The curvature of the fringes below the stress-relief hole is more pronounced on the observed holographic image, and there is an extra elongate fringe on the calculated pattern. Moreover, it was not possible to generate synthetic fringe patterns using the values of stress in better agreement with the measured value of $120 \mathrm{MPa}$, without degrading the match between the observed and calculated fringe patterns.

Several sources of experimental uncertainty, which contribute to the misfit between the observed and calculated fringe patterns in Plate 3, have been identified. These are:

(1) Inadequacy of the simple theory for an infinite plate with a hole. In the laboratory tests a plate of finite extent is used. Hence, the sample has two free boundaries and associated edge effects which are not modelled by the infinite plate theory. Moreover, finite thickness of the plate will have a large effect on the component of displacement normal to the plate surface, in the near vicinity of the hole, as discussed in the previous section. Although the theoretical stress distribution in an infinite plate of finite thickness with a throughgoing circular hole has been described by several authors (e.g. Sternberg \& Sadowsky 1949; Green 1948), no analytic solution exists for the situation appropriate to our laboratory tests; that is, of a shallow hole within a thick plate of finite extent.

(2) The boundary conditions in our laboratory tests are not the same as for the case of an infinite plate. In addition to having two free surfaces, one of the load-bearing surfaces of the sample is at a relatively stiff, nearly fixed boundary (the steel stressing frame), whereas the other load-bearing surface is at a relatively compliant boundary adjacent to the hydraulic jack and steel stress-transmitting blocks. Therefore, the component of displacement $\epsilon_{x}$, parallel to the imposed uniaxial stress $\left(\sigma_{x x}\right)$ is not symmetric about the axis of the cylindrical hole. The appropriate set of boundary conditions is illustrated in Fig. 13. We have attempted to model this effect by setting the strain $\epsilon_{x}$ to zero at the nearly rigid boundary, setting $\epsilon_{x}$ at the compliant boundary as the total strain $\left(\epsilon_{x}\right)$ of the sample in the stress direction, and specifying $\epsilon_{x}$ for intermediate points on the sample surface by linear inter- 


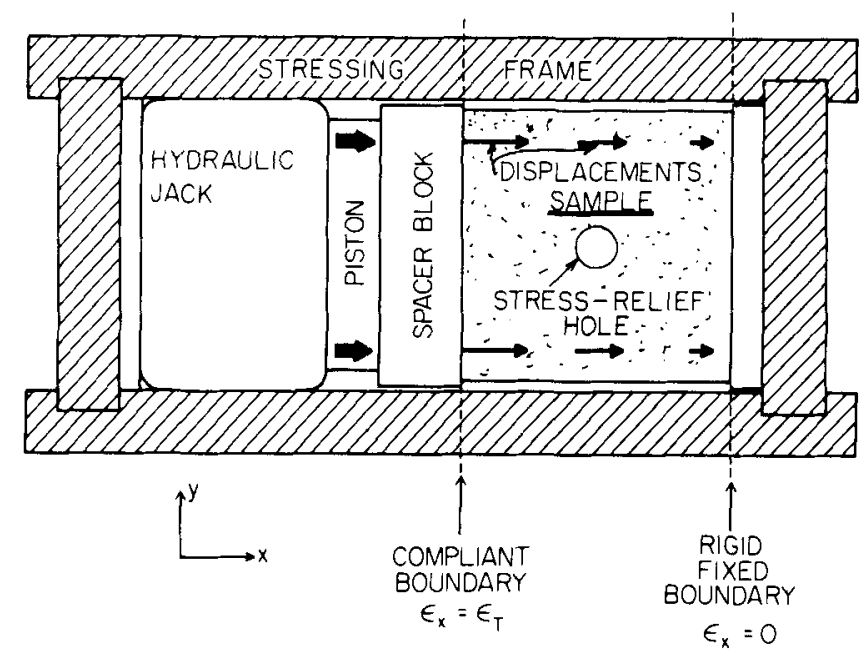

Figure 13. Illustration of the appropriate boundary conditions for our laboratory stress-relief tests. The thick steel side (at right) of the stressing frame is rigid relative to the sample and forms a fixed boundary. Displacements of the sample are accommodated mostly by the piston on the hydraulic jack (left), on which a fairly constant pressure is maintained. Thus, the total strain in the $x$-direction $\epsilon_{t}$, takes place at the piston side of the sample, whereas the strain $\epsilon_{x}=0$ at the rigid boundary. The arrows show displacement decreasing from the compliant to rigid boundaries.

polation. As a result of these boundary conditions, all points on the sample move toward the rigid boundary, and this effect manifests itself on the resulting hologram as a set of elongate fringes subparallel to the rigid boundary. Such a component of motion is clearly seen in Fig. 13. It should be noted that the problem of a 'rigid' and 'compliant' boundary is specific to our laboratory test apparatus, and that this complication is not present in field data obtained in a borehole. Along the same lines, we have assumed in our analysis a constant stress boundary condition at the two load-bearing surfaces, whereas a more appropriate boundary condition might be constant displacement of the load-bearing surfaces. In fact, the constant stress and constant displacement conditions should yield identical results for the strain-relief displacements near the hole, as the size of the plate becomes large compared with the hole diameter.

(3) There may be stress concentrations in the sample due to both the finite size and non-parallelness of the load-bearing surfaces. Stress will be concentrated in those portions of the plate which are wider and contact the stress-transmitting blocks first. This effect may explain, in part, the difference in curvature of the elongate fringes above and below the stress-relieving hole in Plate 3 (top).

(4) Intrinsic uncertainties in the elastic moduli of the sample, the measured value of uniaxial stress, and the geometry of the holographic camera all contribute to the differences between the observed and calculated fringe patterns and stresses.

Considering the approximations in our data analysis procedures and the uncertainties inherent in our laboratory tests, we are encouraged by the level of agreement between the measured $(120 \mathrm{MPa})$ and calculated $(80 \mathrm{MPa})$ stress levels from the example shown in Plate 3. We intend to assess the inadequacies of the infinite plate approximation, and the effect of various boundary conditions, by obtaining numerical solutions for precisely the geometry used in our tests by 3-D finite element analysis. Nevertheless, the results we have obtained so far conclusively demonstrate that we can, in fact, record stress relief fringes in a stressed 


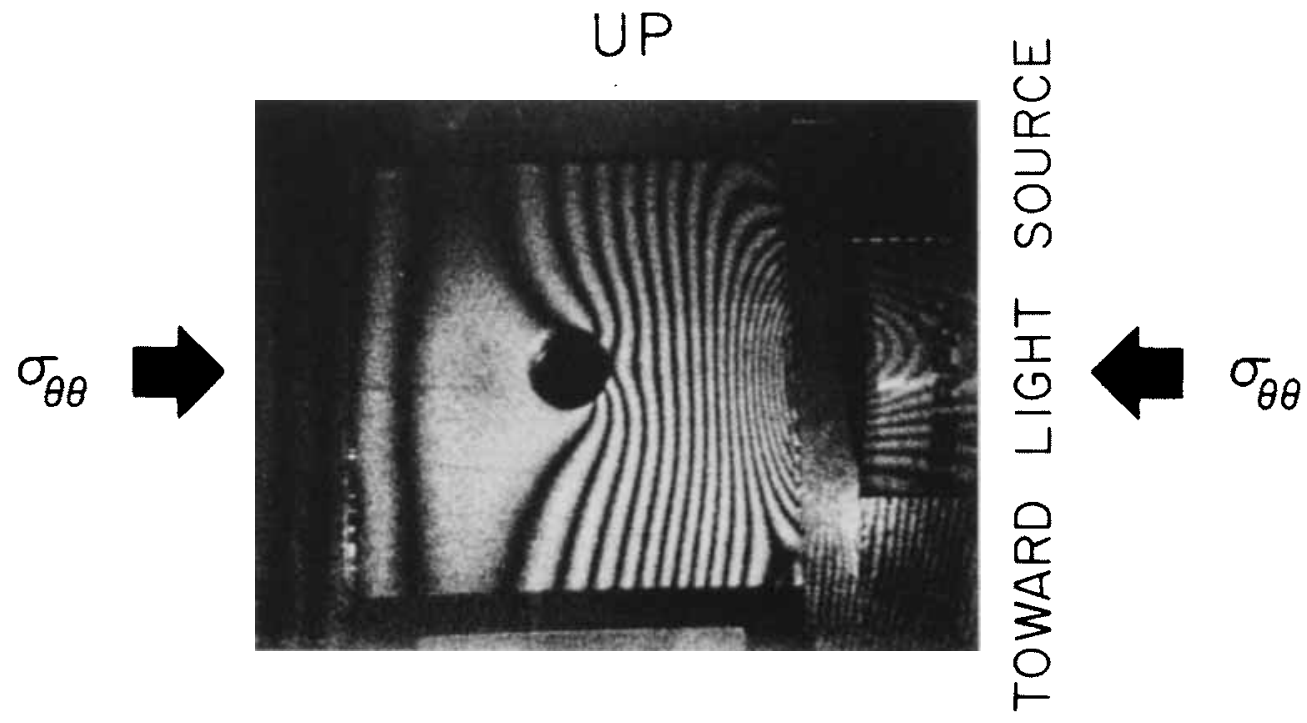

Plate 1. Photograph of a double exposure interference hologram. An aluminium plate with a throughgoing hole is being stressed by a hydraulic jack, seen at the right. In between exposures the pressure on the plate was increased by an amount $\sigma_{\theta \theta}=8.4 \mathrm{MPa}$. Stress concentrations about the advancing piston, as well as perturbations in the stress field due to the hole, are evident in the dark interference fringes on the photograph. 

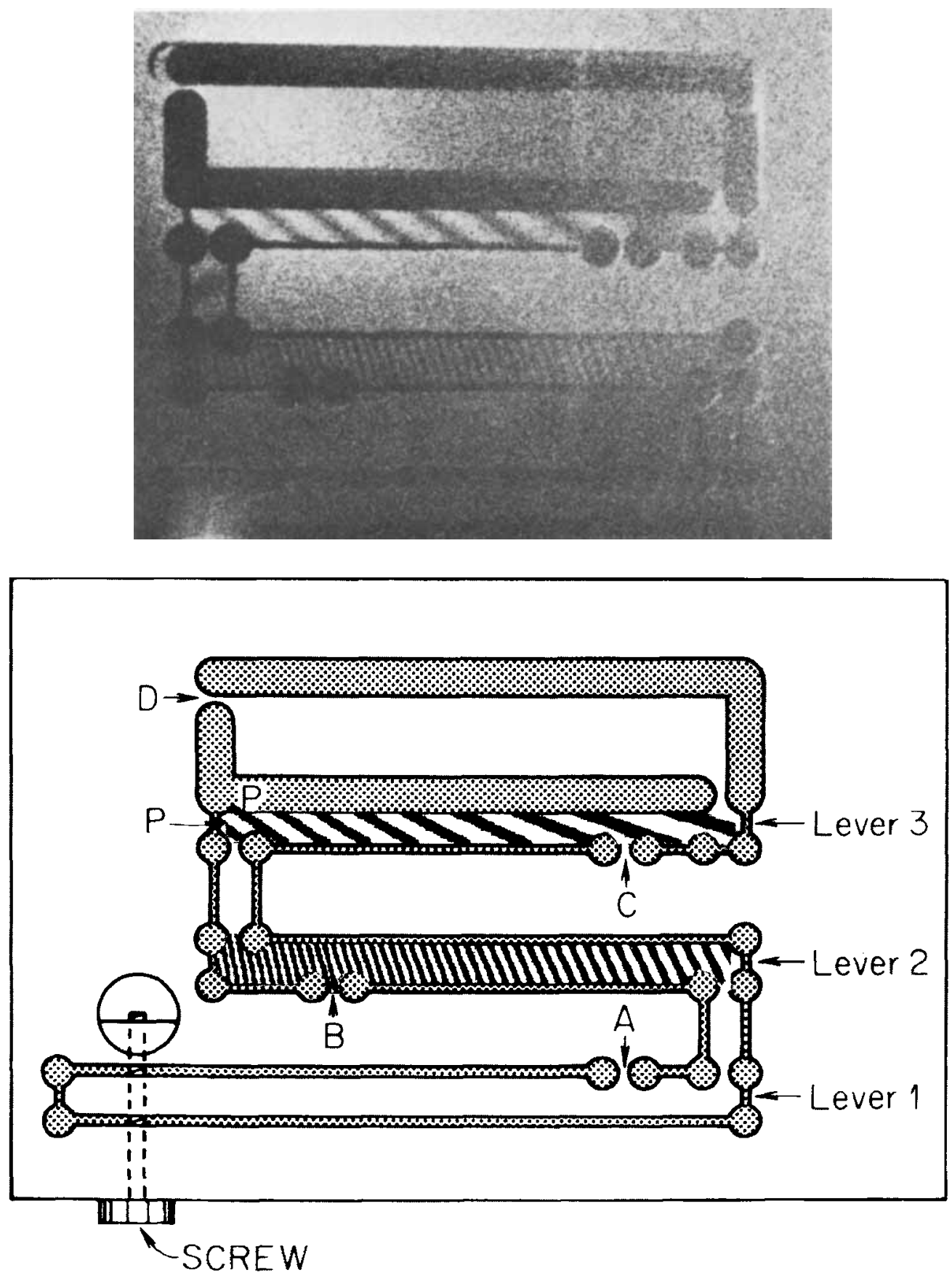

Plate 2. Holographic interferogram of a test object (top) and an illustration of the test object, with calculated interference fringes overlaid (bottom). Lever 1 is displaced by turning a screw, and rotates about hinge point $\mathbf{A}$ (bottom), thus rotating the other levers about their respective hinge points ( $B, C, D)$. The entire assembly is made from a single plate; cutouts are shaded. The displacement of lever 3 was measured at point $P$ as $7.6 \pm 1.2 \mu \mathrm{m}$, whereas the matching calculated fringe pattern indicates a motion of $8.7 \mu \mathrm{m}$ at $\mathrm{P}$. 


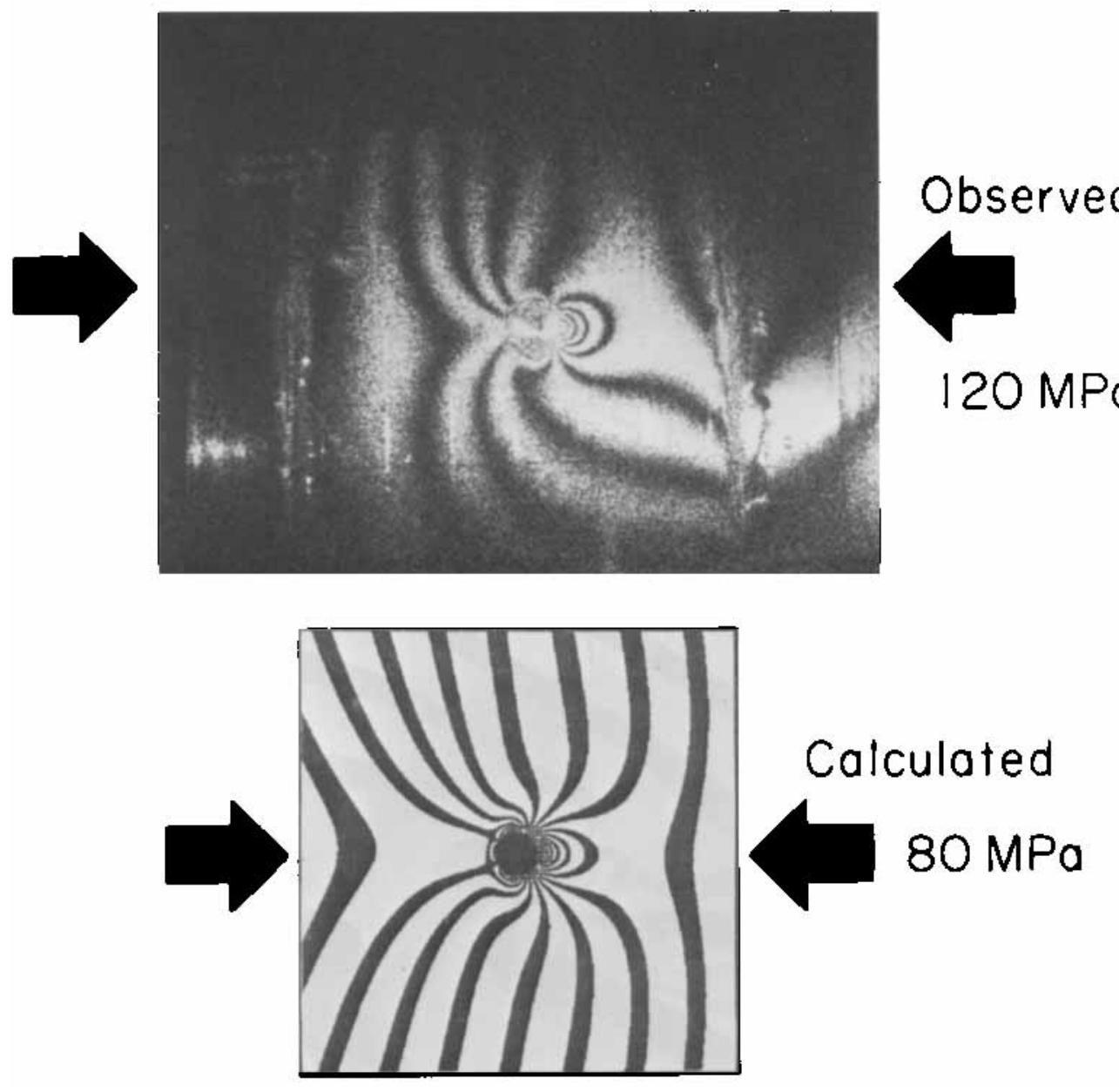

Plate 3. Observed (top) and calculated (bot tom) fringe patterns for stress-relief tests on aluminium 2024 T pre-stressed to a measured value of $120 \mathrm{MPa}$. The stress-relief hole drilled between holographic exposures is $6.25 \mathrm{~mm}$ in diameter. Calculated fringe pattern is based on the plane-stress plate model using a value of $80 \mathrm{MPa}$ uniaxial stress. 
a)

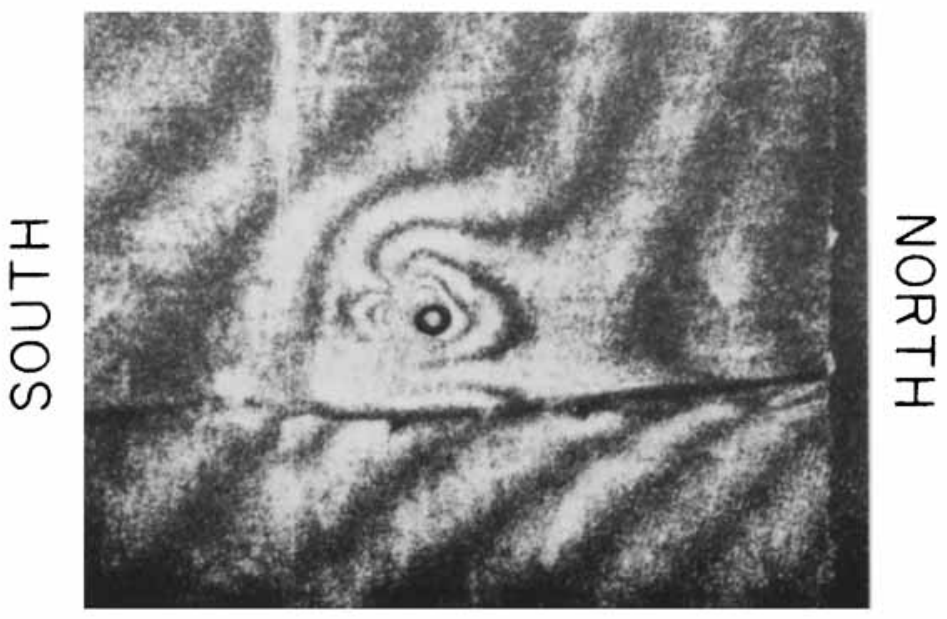

WEST

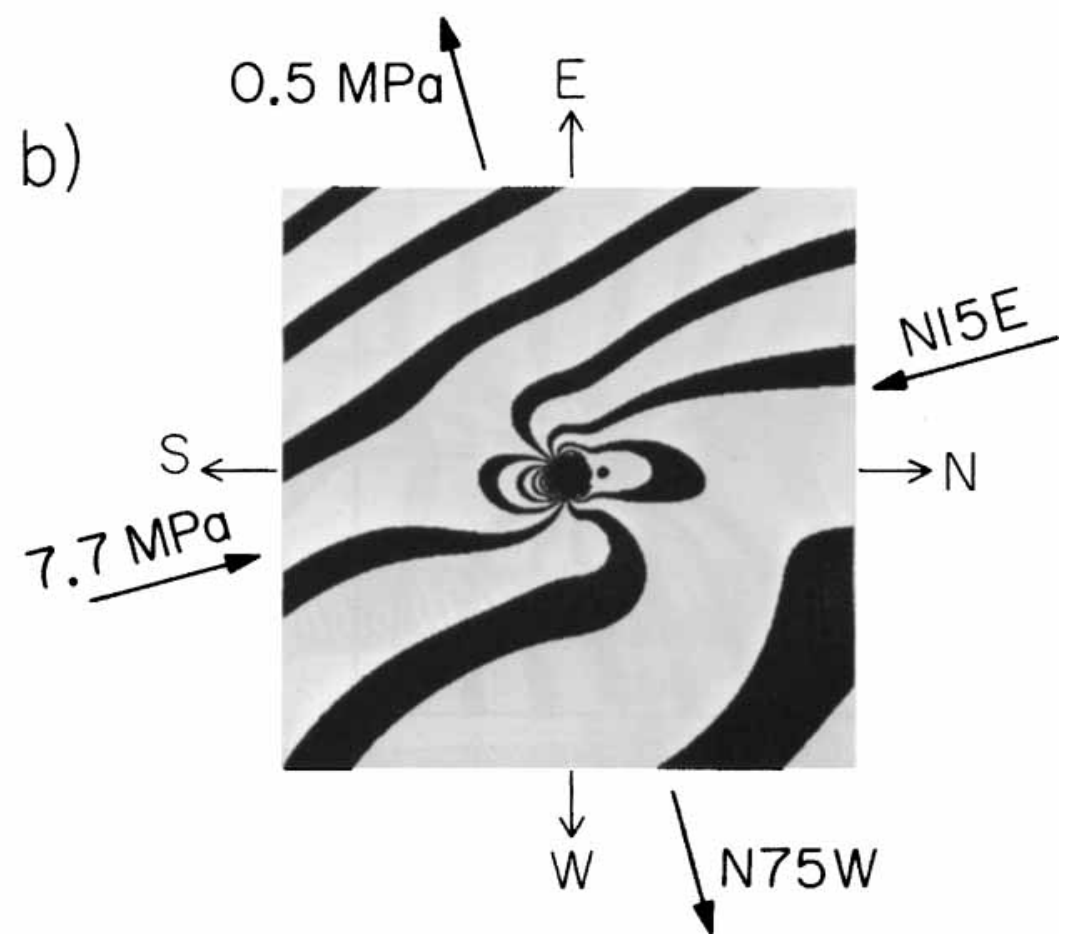

Plate 4. Results of field deployment of the holographic stressmeter in a horizontal borehole. The measurement was made $1.2 \mathrm{~m}$ into the borehole. (a) $1: 1$ photograph of an interference hologram. East is at top and south at left of the photograph. The stress-relief hole is outlined for clarity. Lobed interference fringes near the hole result from stress-relief, whereas the NE-SW subparallel fringes away from the hole are due to slight motion of the instrument. Note the prominent crack, less than $2 \mathrm{~cm}$ from the hole, which greatly complicates interpretation of the data. (b) A theoretical fringe pattern that matches many features of the actual field data in (a). Principal stresses in the plane normal to the stress-relief hole and tangent to the borehole surface ('principal plane stresses') are indicated by heavy arrows; these stresses are appropriate only to the borehole surface and do not represent 'far-field' stresses. The calculations indicate a principal $\mathrm{N} 15^{\circ} \mathrm{E}$ stress of approximately $7.7 \mathrm{MPa}$ parallel to the near face of the pillar, whereas the stress normal to this pillar face has largely been relieved. Note that the hologram view is up towards the surface of the Earth; the photograph is of the borehole 'roof'. 

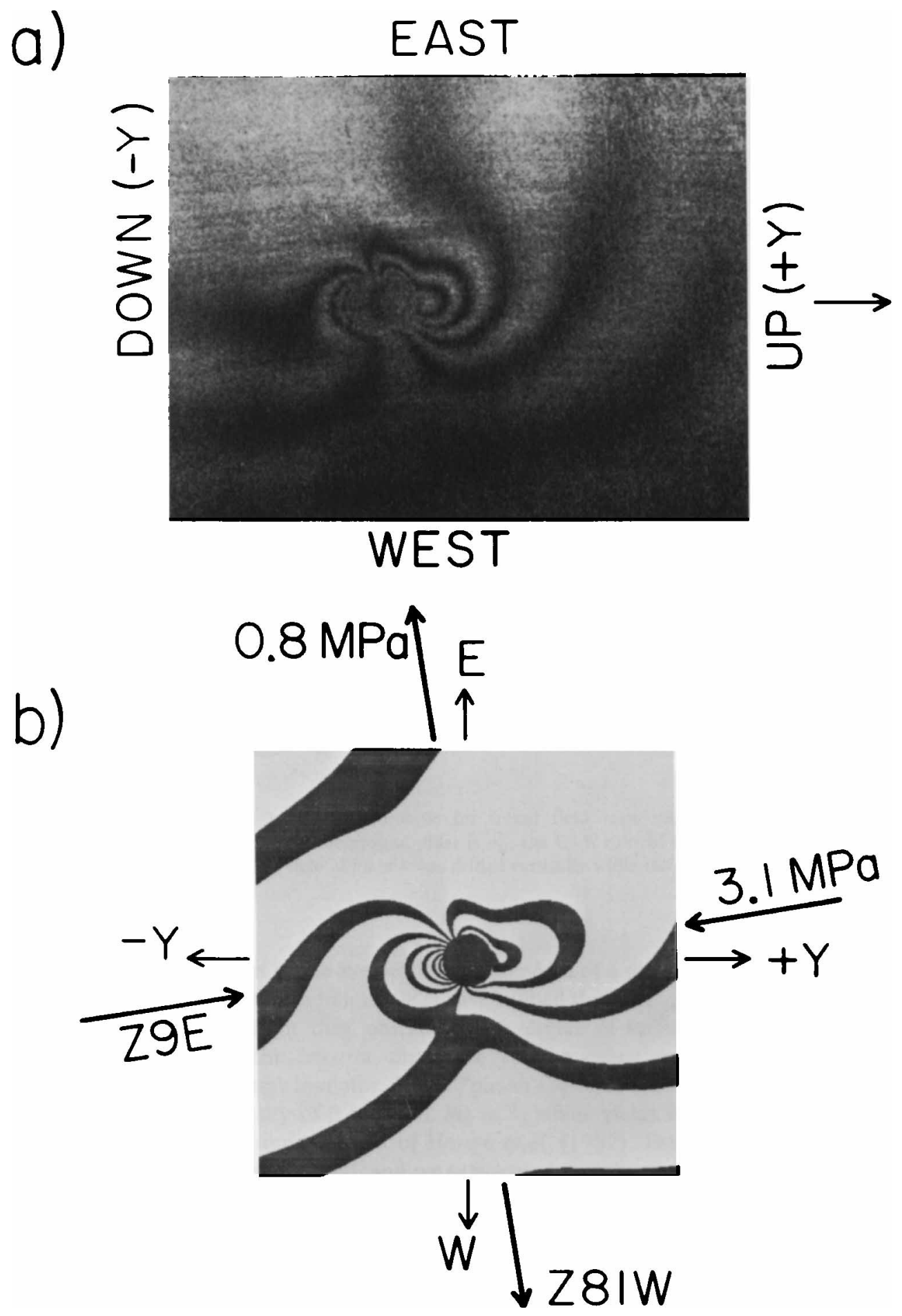

Plate 5. (a) Photograph of an interference hologram taken $3.7 \mathrm{~m}$ in to the same borehole as Plate 4. Stressrelief hole drilled at $90^{\circ}$ from vertical into the south part of the borehole wall has diameter of $0.95 \mathrm{~cm}$ and depth of $1.27 \mathrm{~cm}$. East is at the top side and the surface of the Earth towards the right side of the photograph. (b) Calculated fringe pattern matching the observation in (a). Principal plane borehole stresses are shown. The calculations indicate a near vertical $\mathrm{Y} 9^{\circ} \mathrm{E}$ borehole-wall stress of approximately 3.1 MPa. Note that the hologram view is towards the south (Fig. 14). 
sample, and that from the holographic record of stress relief we can deduce the level of stress on the sample with an uncertainty of about 35 per cent.

\subsection{FIELD RESULTS}

In this section we describe the results of our initial field deployment of the in situ stressmeter. The location of the test site was an underground excavation (180 m overburden) located about $24 \mathrm{~km} \mathrm{NW}$ from the town of Rifle, in Garfield County, Colorado. A horizontal borehole $30.5 \mathrm{~cm}$ in diameter and $7 \mathrm{~m}$ long was drilled for our stress sets into a pillar that measured $15 \mathrm{~m}$ on each side and $8.2 \mathrm{~m}$ high. The sides of the pillar were parallel to the $\mathrm{N}-\mathrm{S}$ and $\mathrm{E}-\mathrm{W}$ directions, and the hole was located $1.8 \mathrm{~m}$ off the floor into the west face of the pillar (see Fig. 14).

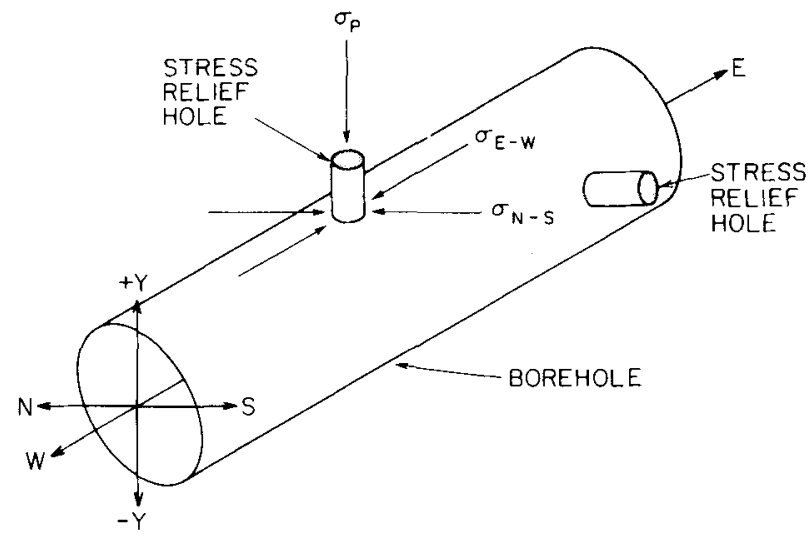

Figure 14. Orientation of the horizontal borehole for initial field tests relative to the geographic directions. The vertical stress in the underground pillar is $\sigma_{p}$, the $\mathrm{E}-\mathrm{W}$ normal stress, $\sigma_{\mathrm{EW}}$, is parallel to the borehole axis. The stress-relief hole of Plate 4 was drilled vertically while the stress-relief hole of Plate 5 was drilled south.

The pillar, as well as most of the overburden, is composed of a shale within the Mahogany formation. Horino, Dolimar \& Tadolini (1982) have studied the mechanical properties of the Mahogany shale and, although they observe a high degree of variability in the physical properties of rock within this horizon, they have found a strong correlation between the shale density and both Young's modulus, $E$, and Poisson's ratio, $\nu$. Our test was performed in shale with a measured density of $2.11 \pm 0.02 \mathrm{Mg} \mathrm{m}^{-3}$, which yields values of $E=8.3 \pm 1.0$ $\mathrm{GPa}$ and $\nu=0.345 \pm 0.05$ from the work of Horino et al. (1982). The pillar, as well as the surrounding rock, was heavily jointed, and we anticipated that the stress distribution within the pillar would therefore be quite complex.

Although the stressmeter was designed for deployment in a vertical borehole, it was adapted for a horizontal hole by attaching a set of ball casters to the instrument, which allowed the stressmeter to be rolled into the hole and rotated. Unfortunately, the initial configuration of casters only allowed effective positioning of the instrument and data collection over a limited angular range of about $\pm 90^{\circ}$ from the vertical position in the borehole. We were not able to obtain sufficient data during our initial field test to constrain all six components of stress independently at a given level in the pillar. However, we successfully fulfilled our immediate goal of observing stress-relief fringes in the field and, in fact, 
obtained sufficient information loosely to constrain the principal stress directions and magnitudes in the underground pillar.

Plates 4 and 5 show the results of two field measurements, as well as synthetic fringe patterns calculated using the infinite plate theory described above. The data displayed in Plate 4 was obtained at a distance of $1.2 \mathrm{~m}$ from the west face of the pillar into the borehole, and that in Plate 5 at a distance of $3.7 \mathrm{~m}$ into the borehole. In Plate 4 the drill was

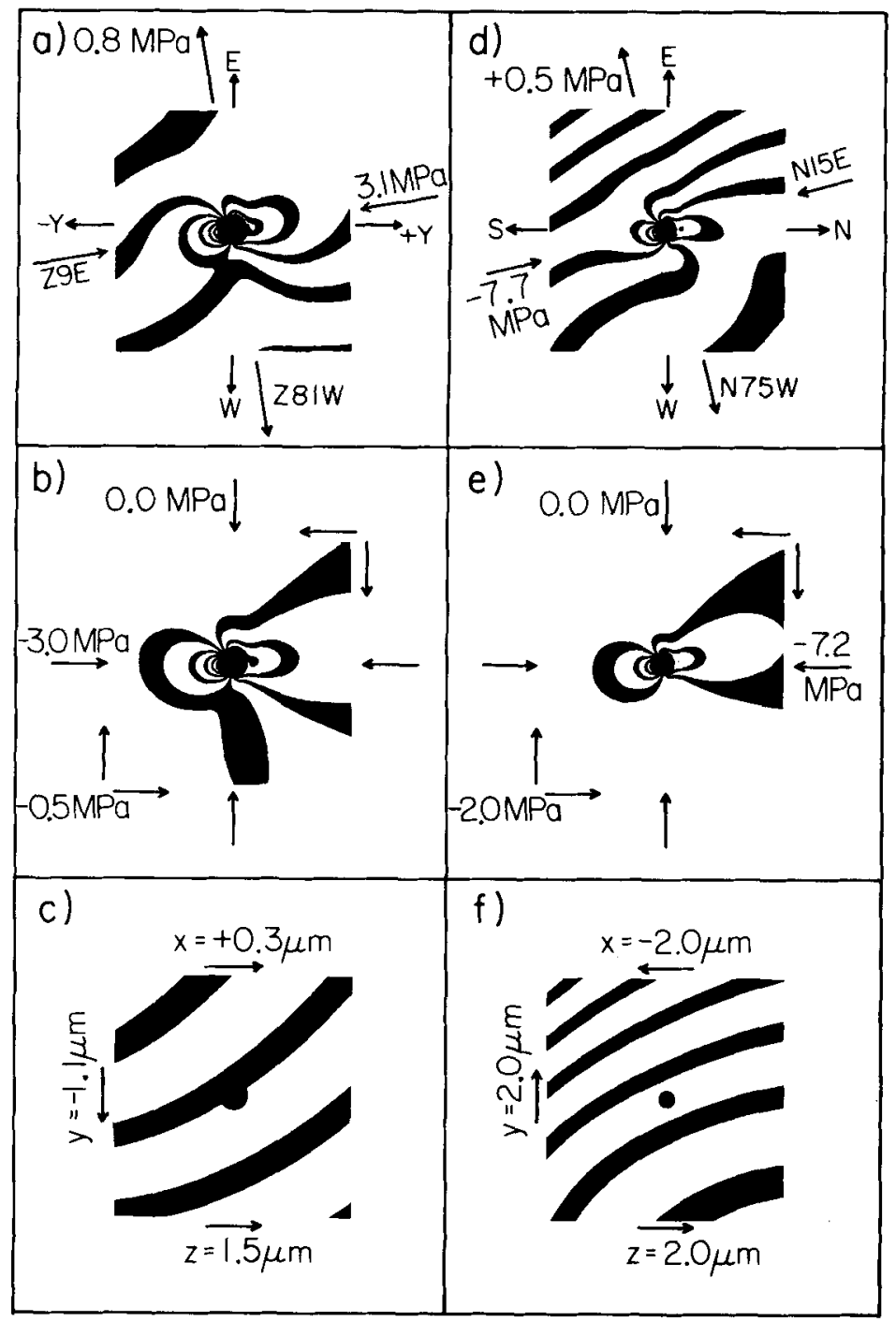

Figure 15. Calculated interference fringe patterns of Plates 4 and 5 are shown in (a) and (d) with principal plane borehole-wall stresses indicated. The fringe patterns of (a) and (d) are the sum of stress-relief displacements of the borehole wall and rigid-body translations of the optics module. Panels (b) and (e) show only the fringe pattern resulting from the stress-relief portion of (a) and (d), respectively. For each, the three non-trivial borehole wall stresses are shown; opposing arrows facing each other indicate a compressive (negative) normal stress and shear stress is indicated by arrows at the corners of the fringe pattern. (c) and (f) indicate the motions (units of $\mu \mathrm{m}$ ) of the optics module for (a) and (d), respectively. Displacements are shown in terms of translation of the borehole wall with respect to the optics module. In both (c) and (f) a positive $z$ translation, toward the stress-relief hole, is indicated. 
facing directly up (vertical), whereas the stress relief hole in Plate 5 was drilled $90^{\circ}$ from the vertical toward the south. Two types of displacement are apparent on each of these photographs. The gently curved subparallel fringes toward the periphery of the photographs are mainly due to a minute rigid body motion of the stressmeter relative to the wall of the borehole, whereas the lobed, sharply curved fringes near the centre of the photographs are dominated by displacements from drilling the stress-relief hole. In Fig. 15 we illustrate the fringe patterns corresponding to these separate components of displacement for the data shown in Plates 4 and 5. All stresses indicated in Plates 4 and 5 and in Figs 15 and 16 were calculated using the theory for a hole in an infinite plate, described earlier, and are therefore stress components within a plane normal to the stress-relief hole and tangent to the surface of the borehole. We refer to the principal components of stress in this plane as the "principal plane borehole stresses' in Plates 4 and 5 and in Figs 15 and 16. Note that these components are appropriate to the stress state at the borehole surface, and not the far-field stress.

A noteworthy feature of Plate 4 is the crack in the borehole. This crack is apparent in the interference hologram as a boundary across which the fringe patterns are displaced, or offset. If the fringe patterns in this interferogram had been due to pure translation or rotation of the stressmeter rather than stress relief of the borehole surface, then each fringe in the pattern would be continuous across the crack. Thus the fringe offset on opposite sides of the crack provides additional evidence that stress relief has indeed been recorded and that the fringe patterns are not merely an artefact. Moreover, these holograms clearly demonstrate the importance of recording the entire displacement field due to stress relief, as opposed to making only local measurements with strain gauges. If a stress-relief hole or slot were made on one side of a crack and a point measurement of displacement were made on the opposite side of the crack, Plate 4 shows that the resultant calculated stress would obviously be in error.

The calculated fringe patterns in Plates 4 and 5 generally provide a good match to the features of the observed fringe patterns, although some differences are apparent. Discrepancies between the observed and calculated patterns arise from a variety of sources: approximation of the borehole surface about the stress-relief hole as an infinite plate, the elastically anisotropic nature of the rock, and the presence of fractures in the rock, to name a few. It is not clear at this point how these various factors affect the actual displacement field or the uncertainties on the stresses calculated using our simple approach. In the future, we will attempt to resolve some of these questions by a numerical finite element analysis of the stress-strain behaviour appropriate to the geometry of our stress-relief experiment. For the present time, we have estimated the uncertainties on the stress levels indicated in Plates 4 and 5 by incrementing the models in stress until the calculated stress patterns are decidedly different from the observed patterns (Fig. 16). On the basis of such calculations, we estimate the uncertainty in our stress determinations to be about \pm 30 per cent in the best case, to 50 per cent in the worst case. This estimate of measurement error does not take into account uncertainties in the moduli of the rock or any systematic bias caused by our modelling procedure. However, it should be noted that additional measurements at other azimuths will constrain measured stress values more tightly and provide a degree of redundancy. Therefore, we estimate that with improvements in the modelling procedure and additional measurements it should be possible in the future to constrain each component of stress with an uncertainty of about $10-20$ per cent.

Two of the stress-relief experiments (Plates 4 and 5) spanning a $90^{\circ}$ azimuth and at nearly a single level in the borehole, yield sufficient information to constrain five of the six independent stress components within the pillar. We need only relate the local stresses at the borehole surface to the 'far-field' stresses. For the purposes of this calculation we define $x$-, 


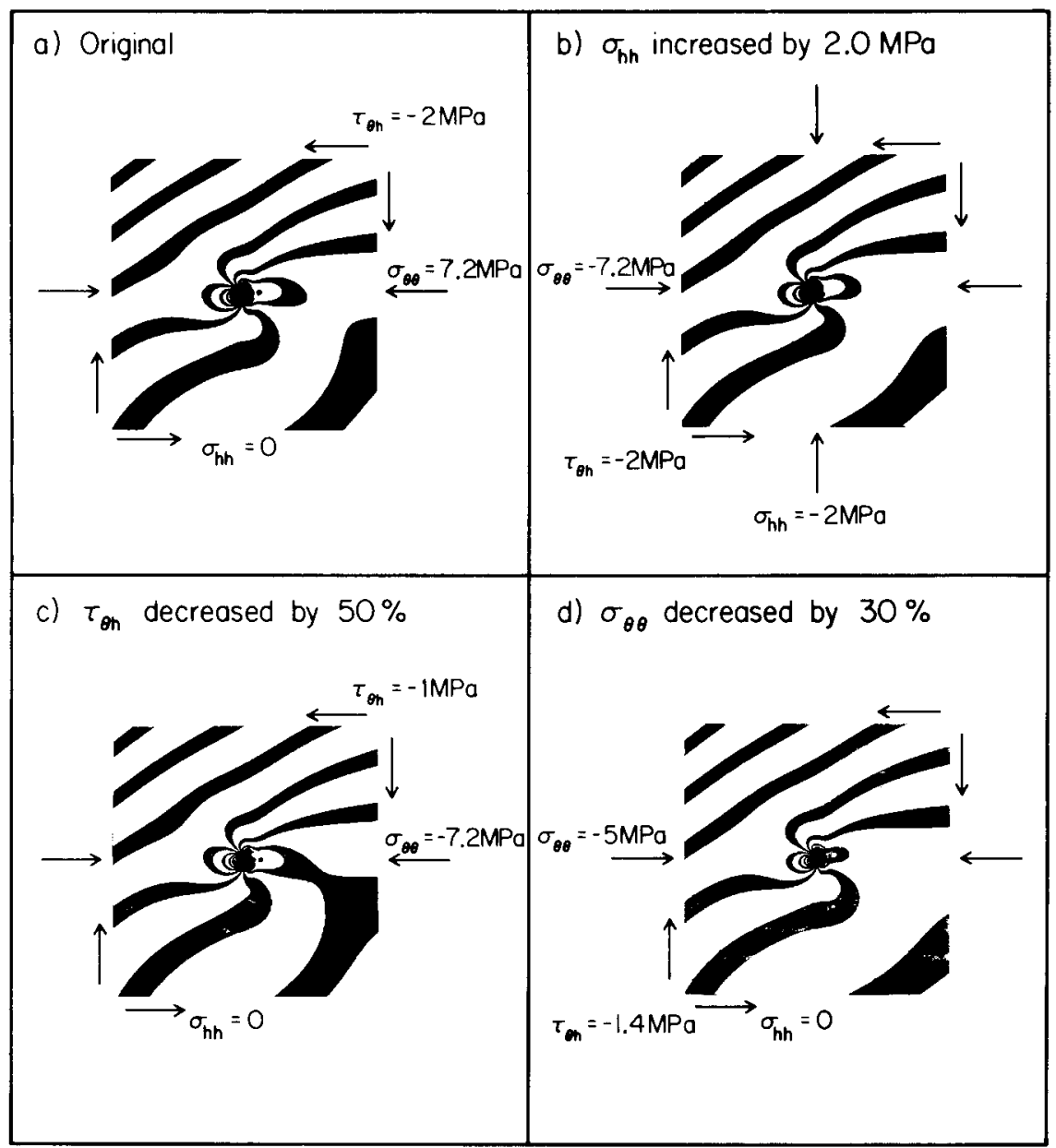

Figure 16. Calculated fringe patterns corresponding to the observed interferogram in Plate 4(a). The magnitude and direction of each stress component is indicated by the arrows (see Fig. 10 for coordinate system). (a) Shows our preferred interpretation of the observed fringe pattern (see Fig. 14b). Panels (b), (c) and (d) show the effect of perturbing the preferred solution for each individual component of stress in (a). Changing the normal stress component $\sigma_{h h}$ from 0 to $-2 \mathrm{MPa}(\mathrm{b})$, results in a discernible but small change in the fringe pattern, indicating that this component is poorly constrained. Perturbations of 50 and 30 per cent for $\tau_{\theta h}$ (c) and $\sigma_{\theta \theta}$ (d), respectively, yield calculated fringe patterns with features not observed in the data, thus giving a measure of the uncertainties in these stress components.

$y$ - and $z$-axes (Fig. 8) coincident with the N-S, vertical and E-W directions, respectively, of Fig. 14. Then the stress-relief experiment of Plate 4 corresponds to an azimuth of $\theta=\pi / 2$, and that of Plate 5 to $\theta=0$ (see Fig. 14). Using the local borehole wall stresses for each case (Fig. 15b, e) in equations (13-18), we calculate the far-field stresses within the pillar listed in Table 1. Calculation of all six independent stress components within the pillar is an underdetermined problem due to lack of data, and $\tau_{\mathrm{NS}-\mathrm{p}}$ is unconstrained. The above results suggest, however, that the maximum principal horizontal stress within the pillar is near a $\mathrm{N}-\mathrm{S}$ orientation.

In a strict sense it is not valid to combine data from two different portions of a pillar to deduce a single stress state. An implicit assumption in this exercise is that the gradient of all 
Table 1. Pillar stresses.

Stress

North-south normal $\sigma_{\mathrm{NS}}$

Vertical normal $\sigma_{\mathrm{p}}$

East-west normal $\sigma_{\mathrm{EW}}$

North-south/vertical shear $\tau_{\text {NS }}$ p

East-west/vertical shear $\tau_{\text {EW-p }}$

North-south/east-west shear $\tau_{\text {NS }}$-EW
Magnitude

(MPa)

$-3.1$

$-2.0$

$-0.7$

Insufficient data

$-0.3$

$+1.0$

* Uncertainties on stress components are generally $\pm 30-50$ per cent) except $\sigma_{\mathrm{EW}}$ (about $\pm 1-2 \mathrm{MPa}$ ). See Fig. 21 .

stress components along the length of the borehole is very small, at least over the distance between our measurements $(2.5 \mathrm{~m}$ or 0.17 times the pillar width). The probability of this condition being satisfied increases with greater distance from the pillar face (Salamon \& Oravecz 1966; Salamon 1974). However, given our limited data base it is not possible at present to assess how good or poor the zero-stress-gradient assumption is, with the single exception of the $\mathrm{E}-\mathrm{W}$ normal component, $\sigma_{\mathrm{EW}}$. Our raw data yield a value of $\sigma_{\mathrm{EW}}=0$, within measurement uncertainty, indicating that any regional or ambient tectonic stress has been fully relieved in the EW direction by excavation. This observation is consistent with the presence of numerous open vertical fractures, such as in Plate 4, which formed in the pillar along pre-existing secondary joints subparallel to the NS direction.

The mean vertical stress in a homogeneous isolated pillar, $\sigma_{\mathrm{p}}$, is commonly obtained by the relation

$\sigma_{\mathrm{p}}=\sigma_{\mathrm{v}}(1-R)^{-1}$

where $\sigma_{\mathrm{v}}(=\rho g h)$ is the vertical stress component in a homogeneous body of rock and $R$ is the areal extraction ratio (Obert \& Duvall 1967). Using $\rho=2.11 \mathrm{~g} \mathrm{~cm}^{-3}$ we calculate an estimated $\sigma_{\mathrm{p}}=-11.2 \mathrm{MPa}$, whereas our holographic measurements yield a vertical stress of $\sigma_{\mathrm{p}}=-2.0 \pm 1 \mathrm{MPa}$, a substantially lower value. However, equation (35) actually places an upper bound on $\sigma_{\mathrm{p}}$. A great variety of factors, including pillar geometry and size, proximity to unmined areas, rock rheology and rock anisotropy can drastically reduce $\sigma_{\mathrm{p}}$, often by a factor of 3 or more from the value predicted by equation (35) (Coates \& Ignatieff 1966; Salamon 1974; Jaeger \& Cook 1979). Moreover, defects such as joints or open fractures, both of which are abundant in our test borehole, may result in local stress values that do not accurately reflect the average stress state. Our measured $\sigma_{p}$ is in fair agreement with the calculated estimate of $\sigma_{v}=-3.8 \mathrm{MPa}$, considering mutual uncertainties in the two values. Given the fact that our test borehole is adjacent (within $15 \mathrm{~m}$, or one pillar width) to an unexcavated portion of the shale seam, our measurement of a low $\sigma_{p}$ would indicate that the vertical pillar stress is close to the virgin overburden pressure, $\sigma_{\mathrm{v}}$. It is also quite possible that the low $\sigma_{\mathrm{p}}$ is purely a local effect.

Bredehoeft et al. (1976) have previously conducted a series of hydrofracture experiments to determine the state of stress in the Piceance Basin, Colorado. These experiments were performed approximately $60 \mathrm{~km} \mathrm{NNW}$ of our initial field deployment site. Bredehoeft et al. found a consistent N70W trend of vertical fractures, and inferred these to be parallel to the maximum principal horizontal stress. At a depth of $\sim 180 \mathrm{~m}$ these authors obtained maximum and minimum horizontal stress levels of approximately -4.8 and $-3.9 \mathrm{MPa}$. For comparison, we obtained horizontal stresses of $-3.1 \mathrm{MPa}$ trending $\mathrm{N}-\mathrm{S}$ and $-0.7 \mathrm{MPa}$ 
trending $\mathrm{E}-\mathrm{W}$. Thus, our measured pillar stresses are consistently lower than measurements made within a continuous rock mass, suggesting a large degree of stress relief in the outer portions of the pillar. At the free surface of the pillar face, the normal stress $\left(\sigma_{\mathrm{EW}}\right)$ should be zero. Our measured value of $\sigma_{\mathrm{EW}}=-0.07$ indicates that nearly complete stress relaxation persists to distances of at least several $\mathrm{m}$ (or 0.2 times the pillar width) away from the pillar face. These results are in agreement with the observations of Van Heerden (1970), who used the 'doorstopper' technique to measure stress in a coal pillar. Van Heerden consistently obtained low and erratic values of horizontal stress, and his values for the ratio of minimum to maximum principal stress brackets our determination. However, near the centre of a pillar the horizontal stresses should attain higher values as the effects of free surfaces are reduced. This effect is clearly seen in our data for the normal component $\sigma_{\mathrm{N}} \mathrm{s}$. The borehole used in the present study is midway between the north and south faces of the pillar and, unlike the situation for $\sigma_{\mathrm{EW}}$, it is in this midplane that maximum values of $\sigma_{\mathrm{NS}}$ are attained. Thus, our apparently large (relative to $\sigma_{\mathrm{EW}}$ and $\sigma_{\mathrm{p}}$ ) value of $\sigma_{\mathrm{NS}}=-3.1 \mathrm{MPa}$ is consistent with the borehole-pillar configuration, and the fact that fractures in the rock have an approximate vertical $\mathrm{N}-\mathrm{S}$ orientation which would least effect stress in the $\mathrm{N}-\mathrm{S}$ direction. Note that the measured $\sigma_{\mathrm{NS}}=-3.1 \mathrm{MPa}$ is in (perhaps fortuitously) close agreement with the value of $-3.9 \mathrm{MPa}$ obtained by Bredehoeft et al. (1976) for the N2OE horizontal component of principal stress. The results of this study are also in qualitative agreement with those of Coates \& Ignatieff (1966), who found the maximum principal pillar stress to be horizontal in a number of cases.

\section{Conclusions}

We have described a practical application of holographic interferometry to record stressrelief displacements in a borehole. A prototype instrument package consisting of a drill to produce a small stress-relief hole, a holographic camera and a laser-light source have been developed for use in deep boreholes. This apparatus can record the entire displacement field about a stress-relief hole drilled into the side of the borehole wall. From holographic displacement measurements at three different azimuths at a given depth in a borehole, and a knowledge of the isotropic elastic moduli of the rock, it is possible to deduce the orientation and magnitude of the three principal far-field stress components acting on the rock without making a priori assumptions about the principal stress directions.

Extensive laboratory calibration tests have been performed with the stress-measuring apparatus. From experiments in which objects have been subjected to simple rigid-body rotations, it was shown that small displacements (less than $10 \mu \mathrm{m}$ ) can be measured with an uncertainty of $\pm 10-15$ per cent. We have also tested the stress-measuring capability of the apparatus in a simulated borehole environment. The major experimental problem in recording displacements by holographic interferometry is to maintain the instrument position to within several micrometres over the course of a measurement. Laboratory tests in an artificial borehole have conclusively demonstrated that this stability criterion is achieved by our stress-measuring apparatus. From these tests, in which the samples consisted of flat rectangular plates under a known uniaxial stress, it was additionally found that the stress state could be measured with an uncertainty of approximately 35 per cent. The fact that this value exceeds the intrinsic experimental uncertainty in displacement measurement by a factor of $2-3$ is ascribed to two effects: (1) we approximate the stress distribution in the samples using the theory for a thin elastic plate of infinite extent with a throughgoing hole; (2) our inability to exactly match the true stress boundary conditions at the edges of the 
sample. These effects may be accounted for and reduced in the future by obtaining the stress distribution numerically using finite-element techniques.

Stress-relief data have been obtained from our initial field deployment of the stressmeter in a horizontal borehole in an oil shale mine. These data establish the viability of holographic interferometry for deducing the level of in situ stress in boreholes. Our results also clearly illustrate the desirability of recording the entire stress-relief displacement field as opposed to making point measurements, for example with strain gauges. The displacement field can be very irregular and complex due to the presence of cracks and other irregularities in the borehole surface, and point displacement measurements can be very misleading. From the initial field experiments, it is possible to constrain five of the six independent components of stress near one face of the pillar. Our results indicate that both the maximum and minimum principal stresses are near horizontal, with the maximum principal (compressive) stress axis trending approximately $\mathrm{N}-\mathrm{S}$. Substantially lower stress levels are deduced in the present study than were measured by Bredehoeft et al. (1976) using hydrofracture techniques, and suggest a large degree of stress relief in the outer region of the pillar. This effect and, perhaps, stress concentrations in untested portions of the pillar, apparently overwhelms any residual imprint of the far-field tectonic stress. Additional data obtained in a borehole farther removed from the effects of underground excavation would be required in making any further comparisons.

Although our results have demonstrated the viability of performing in situ stress-relief measurements by holographic interferometry, the present $30 \mathrm{~cm}$ diameter instrument is too large for deployment in most scientific boreholes. Based upon experience gained with the prototype instrument we are currently designing a smaller apparatus with a diameter of less than $15 \mathrm{~cm}$. In parallel with this instrumental work we are developing data reduction procedures to invert holographic interferograms for stress-relief displacements on the borehole surface, in a way similar to the method of Dhir \& Sikora (1972). This method is less direct than the forward modelling approach utilized in the present work, but should provide unique and more precise displacement values and will allow the application of standard statistical methods to assign uncertainties to the solutions. A related matter is to obtain a more realistic mathematical model of the borehole wall response to drilling a stress-relief hole. The results of our work to date indicate that although the elastic plate theory can explain many features observed on the holographic interference patterns, a precise description of the ambient stress field in the borehole from the holographic data requires a closer approximation to the actual borehole geometry. Future data reduction efforts will employ numerical finite element methods to model the displacements resulting from a finite depth stress-relief hole and account for stress gradients about the borehole circumference.

\section{Acknowledgments}

We thank J. Schmidt, D. Tomren, D. Evensen, P. Bhuta and S. N. Cohn for their efforts in the design of earlier versions of the holographic stressmeter. Numerous components of the instrument were designed in collaboration with B. Barber, W. Ginn and W. Miller. C. Hudson, R. Wickes and B. Barber fabricated and repaired innumerable parts with a great deal of skill and a surprising amount of cheer. Discussions with R. Wuerker, L. Heflinger, B. Hager, T. Webb, A. S. Nieto and A. Frankel were of immense value and are greatly appreciated as is the careful review of T. Engelder. We gratefully acknowledge support of this research by the US Department of Energy, Contract number DE-ATO3-83ER13120, and gifts from Schlum. berger Technology Corporation and Sun Oil Company. Contribution 4121 of the Division of Geological and Planetary Sciences, California Institute of Technology, Pasadena, California. 


\section{References}

Ahrens, T. J., Jacoby, J. L. \& Bhuta, P. G., 1975. A holographic instrument for in situ stress measurement in deep boreholes, Trans. Am. Geophys. Un., 56, 244.

Aleksandrov, E. B. \& Bonch-Bruevich, A. M., 1967. Investigation of surface strains by the hologram technique, Soviet Phys., Techn. Phys., 12, 258-265.

Bock, H., Foruria, V.\& Lequerica, R., 1984. A new stress relief concept for in situ stress measurements in rock and its implementation in two recoverable stressmeters, in Fourth Australia-New Zealand Conference on Geomechanics, Per th, Australia, May 14-18.

Bredehoeft, J. D., Wolff, R. G., Keys, W. S. \& Shuter, E., 1976. Hydraulic fracturing to determine the regional in situ stress field, Piceance Basin, Colorado, Bull. geol. Soc. Am., 87, 250-258.

Coates, D. F. \& Ignatieff, A., 1966. Prediction and measurement of pillar stresses, Can. Min. J., 87, 5056.

Cohn, S. N., 1983. Holographic in-situ stress measurement in geophysics, PhD thesis, California Institute of Technology.

Cohn, S. N. \& Ahrens, T. J., 1982. In-situ stress measurement with holographic stressmeter: field deployment and control experiments, Eos, Trans. Am. Geophys. Un., 45, 1111.

Dhir, S. K. \& Sikora, J. P., 1972. An improved method for obtaining the general displacement field from a holographic interferogram, Exp. Mech., 12, 323-327.

Doe, T. W. Hustrulid, W. A., Leijon, B., Ingvold, K. \& Strindell, L., 1983. Determination of the state of stress at the Stripa Mine, Sweden, in Hydraulic Fracturing Stress Measurements, pp. 119-129. eds Zoback, M. D. \& Haimson, B. C., US National Academy of Sciences.

Fairhurst, C., 1964. Measurement of in-situ rock stresses with particular reference to hydraulic fracturing, Felsmech. Ingenieurgeol., 2, 129-147.

Gabor, D., 1948. A new microscopic principle, Nature, 161, 777-778.

Gabor, D., 1949. Microscopy by reconstructed wavefronts, Proc. R. Soc., A, 197, 454-487.

Gabor, D., 1951. Microscopy of reconstructed wavefronts II, Proc. Phys. Soc., 64, 449-469.

Granryd, L., Getting, I. C. \& Spetzler, H., 1983. Path dependence of acoustic velocity and attenuation in experimentally deformed Westerly granite, Geophys. Res. Lett., 10,71-74.

Green, A. E., 1948. Three-dimensional stress systems in isotropic plates. I, Trans. R. Soc. A., 240, 561597.

Hager, B. H. \& O'Connell, R. J., 1981. A simple global model of plate dynamics and mantle convection, J. geophys, Res., $86,4843-4867$.

Haimson, B. C., 1975. The state of stress in the earth's crust, Rev. Geophys. Space Phys., 13, 350-352.

Haimson, B., 1977. Crustal stress in the continental United States as derived from hydrofracturing tests, Geophys. Monogr. 20, The Earth's Crust, pp. 576-592, American Geophysical Union, Washington, DC.

Haines, K. A. \& Hildebrand, B. P., 1966. Surface-deformation measurement using the wavefront reconstruction technique, Appl. Opt., 5, 595-602.

Heflinger, L. O., Wuerker, R. F. \& Brooks, R. E., 1966. Holographic interferometry, J. appl. Phys., 37, 642-649.

Hiramatsu, Y. \& Oka, Y., 1962. Stress around a shaft or level excavated in ground with a threedimensional stress state, Mem. Fac. Engng, Kyoto, 24, 56-76.

Horino, F. G., Dolinar, D. R. \& Tadolini, S.C., 1982. Mechanical properties of the mahogany zone shale, Garfield County, Colorado, US Bureau of Mines Internal Report, United States Department of the Interior, Bureau of Mines, Denver, Colorado.

Horman, M. H., 1965. An application of wavefront reconstruction to interferometry, Appl. Opt., 4, 333-336.

Jaeger, J. C. \& Cook, N. G. W., 1979. Fundamentals of Rock Mechanics, Chapman \& Hall, London.

Kurita, K., Swanson, P. L., Getting, I. C. \& Spetzler, H., 1983. Surface deformation of Westerly granite during creep, Geophys. Res. Lett., 10,75-78.

Leeman, E. R. \& Hayes, D. J., 1966. A technique for determining the complete state of stress in rock using a single borehole, Proc. 1st Congr. int. Soc. Rock Mech. Lisbon, 2, 17-24.

Leith, E. M. \& Upatnieks, J., 1962. Reconstructed wavefronts and communication theory, J. opt. Soc. Am., 52, 1123-1130.

Leith, E. M. \& Upatnieks, J., 1964. Wavefront reconstruction with diffused illumination and threedimensional objects, J. opt. Soc. Am., 54, $1295-1301$.

Lyman, T. (ed.), 1971. Metals Handbook, 8th edn, 1, American Society for Metals. 
McGarr, A. \& Gay, N. C., 1978. State of stress in the Earth's crust, Ann. Rev. Earth planet. Sci., 6, 405436.

McGarr, A., Zoback, M. D. \& Hanks, T. C., 1982. Implications of an elastic analysis of in-situ stress measurements near the San Andreas fault, J. geophys. Res., 87, 7797-7806.

Nakamura, K. \& Uyeda, S., 1980. Stress gradient in arc-back arc regions and plate subduction, J. geophys. Res., 85, 6419-6428.

Obert, L. \& Duvall, W. I., 1967. Rock Mechanics and the Design of Structures in Rock, Wiley, New York.

Powell, R. L. \& Stetson, K. A., 1965. Interferometric vibration analysis by wavefront reconstruction, J. opt. Soc. Am., 55, 1593-1598.

Richardson, R. M., Solomon, S. C. \& Sleep, N. H., 1979. Tectonic stress in the plates, Rev. Geophys. Space Phys., 17, 981-1019.

Salamon, M. D. G., 1974. Rock mechanics of underground excavations, in Advances in Rock Mechanics, Proc. 3rd Cong. int. Soc. Rock Mech., IB, 951-1099, National Academy of Sciences, Washington, DC.

Salamon, M. D. G. \& Oravecz, K. I. 1966. Displacement and strains induced by bord and pillar mining in South African collieries, Proc. 1st Cong. int. Soc. Rock Mech., Lisbon, 2, 227-231.

Schmidt, J. L., Ahrens, T. J., Jacoby, J. L., Tomren, D. R., Evensen, D. A. \& Bhuta, P. G., 1974. An optical instrument for in-situ stress measurements in rocks, TRW Rep. AT-SVD-TR-74-6.

Simonsen, E. R., Abou-Sayed, A. S. \& Clifton, R. J., 1978. Containment of massive hydraulic fractures, Soc. Petrol Eng., 18.

Smith, R. L. \& Bailey, R. A., 1968. Resurgent cauldrons, Mem. geol. Soc. Am., 116, 613-662.

Sollid, J. E., 1969a. Translational displacements versus deformation displacements in double exposure holographic interferometry, Opt. Communs., 2, 282-288.

Solidd, J. E., 1969b. Holographic interferometry applied to measurements of small static displacements of diffusely reflecting surfaces, Appl. Opt., 8, 1587-1595.

Solomon, S. C., Richardson, R. M. \& Bergman, E. A., 1980. Tectonic stress: models and magnitudes, J. geophys. Res., 85, 6086-6092.

Spetzler, H. A., Getting, I. C. \& Martin, R. J., 1979. Application of holographic interferometry to high pressure experiments, in High Pressure Science and Technology, Vol. 1, eds Timmerhaus, K. D. \& Barber, M. S., Plenum, New York.

Spetzler, H. \& Martin, R. J. (III), 1974. Correlation of strain and velocity during dilatancy, Nature, 262, $30-31$.

Sternberg, E. \& Sadowsky, M. A., 1949. Three-dimensional solution for the stress concentration around a circular hole in a plate of arbitrary thickness, J. appl. Mech., 16, 27-38.

Timoshenko, S. P. \& Goodier, J. N., 1970. Theory of Elasticity, McGraw-Hill, New York.

Van Heerden, W. L., 1970. Stress measurements in coal pillars. Proc. 2nd Congr. int. Soc. Rock Mech., Belgrade, 2, 497-501.

Vest, C. M., 1979. Holographic Interferometry, Wiley, New York.

Waters, J. P., 1974. Holography, in Holographic Nondestructive Testing, pp. 5-59, ed. Erf, R. K., Academic Press, New York.

Zoback, M. D.\& Haimson, B. C., 1983. Hydraulic Fracturing Stress Measurements, US National Academy of Sciences.

Zoback, M. D., Tsukahara, H. \& Hickman, S., 1980. Stress measurements at depth in the vicinity of the San Andreas Fault: implications for the magnitude of shear stress at depth, J. geophys. Res., 10 , $6157-6173$. 